\title{
Frequency domain description of Kohlrausch response through a pair of Havriliak-Negami-type functions: An analysis of functional proximity
}

\author{
J. S. Medina,,${ }^{1,}$ R. Prosmiti,${ }^{1}$ P. Villarreal,,${ }^{1}$ G. Delgado-Barrio, ${ }^{1}$ and J. V. Alemán ${ }^{2}$ \\ ${ }^{1}$ Instituto de Física Fundamental, IFF-CSIC, Serrano 123, Madrid ES-28006, Spain \\ ${ }^{2}$ Departamento Química, Facultad de Ciencias del Mar, ULPGC, Campus Universitario de Tafira, Las Palmas de G. Canaria ES-35017, Spain
}

(Received 4 July 2011; revised manuscript received 10 October 2011; published 16 December 2011)

\begin{abstract}
An approximation to the Fourier transform (FT) of the Kohlrausch function (stretched exponential) with shape parameter $0<\beta \leqslant 1$ is presented by using Havriliak-Negami-like functions. Mathematical expressions to fit their parameters $\alpha, \gamma$, and $\tau$, as functions of $\beta(0<\beta \leqslant 1$ and $1<\beta<2)$ are given, which allows a quick identification in the frequency domain of the corresponding shape factor $\beta$. Reconstruction via fast Fourier transform of frequency approximants to time domain are shown as good substitutes in short times though biased in long ones (increasing discrepancies as $\beta \rightarrow 1$ ). The method is proposed as a template to commute time and frequency domains when analyzing complex data. Such a strategy facilitates intensive algorithmic search of parameters while adjusting the data of one or several Kohlrausch-Williams-Watts relaxations.
\end{abstract}

DOI: 10.1103/PhysRevE.84.066703

\section{INTRODUCTION}

\section{A. Ubiquity and relationship to other relaxations}

The stretched exponential function, or Kohlrausch function $\phi_{K, \beta}(t) \equiv e^{-t^{\beta}}, 0 \leqslant t<\infty, 0<\beta \leqslant 1[1]$, is one of most widely used relaxation functions to describe retarded (step) response in the temporal domain (henceforth called $t$ space) when talking about polymer dielectric spectroscopy, photoluminiscence, capacitor discharging, elasticity stress relaxation, $\alpha$ transition in vitreous or glassy materials [2-5], etc. There is no doubt, when looking at its geometrical shape, at the Cole-Cole diagram in frequency space $(\omega$ space), that it should belong, though distorted, to a more spacious family of relaxation functions which describe such physical behavior of loss and reaction in the areas cited. The most common and simple exponents of this family would be the Debye (D) [6], $\frac{1}{1+i \omega}$; the Cole-Cole (CC) [7], $\frac{1}{1+(i \omega)^{\alpha}}, 0<\alpha<1$; the Cole-Davidson (CD) [8], $\frac{1}{(1+i \omega)^{\gamma}}, 0<\gamma<1$; and the Havriliak-Negami (HN) [9], $\frac{1}{\left(1+(i \omega)^{\alpha}\right)^{\gamma}}, 0<\alpha<1,0<\gamma<1$, functions. ${ }^{1}$ Nevertheless the expression of $\phi_{K, \beta}$ in the $\omega$ space is nearly always in the form of an asymptotic or slow convergence series depending on values of $\beta$ and $\omega$ [10-15], or numerically calculated [4,16-19], which makes it difficult to establish and quantify a "functional proximity" of physical meaning to the above-mentioned functions. In the same way the relaxation functions in Fourier space or it is not possible to express them in a compact form (HN), or they exhibit unphysical properties, as a divergence at $t=0$, when described in $t$ space (CD) [20].

Despite the anomalies, great efforts are made to describe the Kohlrausch function as a numerical table, an algorithm, or an approximation as a sum of related functions in $\omega$ space because of its ubiquity and importance in condensed matter physical phenomena $[4,17,19,21-23]$. In addition the behavior in $\omega$ space is expected to be more regular than that in $t$ space, though the latter is thought of as more natural

\footnotetext{
*tlazcala@yahoo.es

${ }^{1}$ See Appendix A.
}

PACS number(s): 07.05.Kf, 02.30.Gp, 61.20.Lc, 02.30.Uu

to a physical description of molecular dynamics, e.g., the Kohlrausch relaxation function as a consequence of multiscale cluster relaxation or a paused or frozen random walk of dipolar orientations [24,25]. Moreover, in conjunction with the mathematical uniformity, which allows an easier computational treatment, some light is shed on the structure in experimental terms. Since the Kohlrausch function is expressed as a "sum" of exponential relaxations weighted in their characteristic times (i.e., the Laplace transform of a positive density $g_{\beta}(s) \geqslant 0$ when $0<\beta \leqslant 1)[13,16,26-32]$ its counterpart in $\omega$ space is expressible too as a weighted "sum" of simple and regular relaxations of Debye type (i.e., the Stieltjes transformation minus the same density $\left.g_{\beta}(s)[29,33,34]\right)$.

This decomposition allows us to interpret the global relaxation in $\omega$ space as the one of small parts in a larger superstructure, for example, simple dipolar relaxation of polar residues in a larger polymeric molecule or that of clusters with a distribution of scales among them [3-5,24,25]. Of course this physical insight serves as a mathematical description but requires a deeper analysis of every system to offer an explanatory mechanism. In addition to the Kohlrausch relaxation function, others are used with the same purpose and techniques (D, CC, CD, HN, and some mixed types), whereby a primary functional form to generate the others should be chosen on the basis of mathematical or computational properties instead of simple physical interpretation [20,35].

The Kohlrausch function shows some unphysical characteristics at early times $[4,23,36]$; for any $0<\beta<1$ the slope of the function at $t=0$ is infinity. So though the collective (correlated) motion of the ensemble is well described at large $t$, the inertia of individual elements has been usually disregarded in the relaxation function at small times. At large times the function is however appropriate [3] and a displacement in time origin could avoid the initial singularity [4,23]. Consequently the indicated correction solves the problem in $t$ space at the expense of complicating even more an expression in $\omega$ space. This drawback forces two considerations about the change from a temporal domain to a frequency one through one-sided Fourier transform. One is that any physically based modification of Kohlrausch's law must be accompanied by an efficient algorithm to calculate Fourier coefficients of the 
original function as they will be the first-order approximation to the new ones and the starting point for a series expansion. No specific clues in the literature have pointed out how to do this [4,23]. Second, we should note that most likely the analytical function represented by the series and corresponding to Kohlrausch's law in $\omega$ space possesses mathematical singularities we overlooked at first.

This latter point dissuades one from a direct approach over Fourier transform of the Kohlrausch function expressed as a $\omega$ series (see Sec. II A). So in Sec. II we examine some characteristics of the Kohlrausch function and its derivative in $t$ space and $\omega$ space, which sustain the election of this derivative as the function to be handled. In Sec. III our approximation is presented, and in Sec. IV departure (error) of the reconstruction from the original function, as well as the possible extension to values of the squeezed exponential, $1<\beta<2$, not so common in the literature $[19,32]$, are discussed.

\section{REGULARITY IN THE FOURIER TRANSFORM}

\section{A. Mathematical considerations}

Considering $\omega \in \mathbb{C}$, any singularity outside of the real line $(\omega \in \mathbb{R})$ will restrict the convergence of the Fourier series and its numerical usefulness $(\omega \rightarrow 0$ implies large oscillating terms [10,30-32]). In this sense Wintner [10] has shown that

$$
\begin{aligned}
\omega \int_{0}^{\infty} e^{-t^{\beta}} \cos (\omega t) d t & =\omega^{-\beta} \int_{0}^{\infty} e^{-t \omega^{-\beta}} \sin \left(t^{\frac{1}{\beta}}\right) d t \\
& \equiv \omega^{-\beta} \varpi_{\beta}\left(\omega^{-\beta}\right),
\end{aligned}
$$

with $\omega>0$ and $\omega^{-\beta}>0$, and proved that $\varpi_{\beta}(z), z \in \mathbb{C}, 0<$ $\beta<1$, is an entire function. The problem appears when doing the transformation backward because it has branch points (rational or transcendental) and it is singular at $\omega=0$, which destroys for the two-sided Fourier transform the analytical properties obtained for $\varpi_{\beta}(z)$. The Taylor expansion of $\varpi_{\beta}(z)$ gives the series for the Fourier transform and explains why it converges for $\beta<1$ and diverges for $\beta>1$, the latter being useful only as an asymptotic expansion.

Thus a direct approach in calculating Kohlraush's law Fourier transform is not necessarily the best mathematical option, nor is a physical approach because in many experiments the relaxation function is not measured, but the normalized transient function is measured instead. This one, for example, in experiments with dielectrics, is obtained when a constant electric field polarizing a sample is switched off instantaneously and the induced field is fading off. In terms of frequency response we write the complex dielectric constant, $\varepsilon^{*}(\omega)$, as

$$
\frac{\varepsilon^{*}(\omega)-\varepsilon_{\infty}}{\varepsilon_{o}-\varepsilon_{\infty}}=\int_{0}^{\infty} e^{-i \omega t}\left(-\frac{d \phi}{d t}\right) d t
$$

so it makes sense to describe the derivative $-\phi^{\prime}(t)$ and integrate it later to recover the response function. Besides it has the advantage of our ability to modify it parametrically to represent a wider kind of relaxations starting from that of Kohlrausch and ending in a CD function [20].

According to such mathematical and physical reasons, we are prone to expect a nonsingular approximation through a detailed description of the first derivative. To strengthen this point of view, we review the analytical behavior of both the function and its derivative in the $t$ and $\omega$ domains.

\section{B. The extended exponential function: $e^{-t^{\beta}}$}

We denote the one-sided Fourier transform of $e^{-t^{\beta}}$ as

$$
\chi_{\beta}(\omega)=\int_{-\infty}^{\infty} H(t) e^{-t^{\beta}-i \omega t} d t=\int_{0}^{\infty} e^{-t^{\beta}-i \omega t} d t
$$

and the transform of its (minus) derivative as

$$
\begin{aligned}
\psi_{\beta}(\omega) & =\int_{-\infty}^{\infty} H(t) \beta t^{\beta-1} e^{-t^{\beta}-i \omega t} d t \\
& =\int_{0}^{\infty} \beta t^{\beta-1} e^{-t^{\beta}-i \omega t} d t,
\end{aligned}
$$

with $H(t)$ being the Heaviside function. Integrating by parts and taking into account that $\phi_{K, \beta}(0)=1, \phi_{K, \beta}(t \rightarrow \infty)=$ 0 , and $\left|e^{-i \omega t}\right|=1 \forall t \in \mathbb{R}$, we obtain the following closure relation:

$$
1=-\int_{0}^{\infty} \frac{d}{d t}\left[\phi_{K, \beta}(t) e^{-i \omega t}\right] d t=\psi_{\beta}(\omega)+i \omega \chi_{\beta}(\omega) .
$$

Any approximation to $\psi_{\beta}(\omega)$ will give us automatically another one to $\chi_{\beta}(\omega)$ allowing the latter to recover, by inverse transform, a function close to $e^{-t^{\beta}}$. So the proximity obtained in reconstruction in $t$ space will grade the success achieved in mimicking $\psi_{\beta}$. In this sense we should point out a handicap always present independently of goodness of numerical packages utilized (e.g., fftw3 for Fourier Transform [37]): along with the discretization of the integrals implicit in the fast Fourier transform (FFT) algorithm and the sampling chosen to represent $\phi_{K, \beta}\left(t_{i}\right)$ and $-\phi_{K, \beta}^{\prime}\left(t_{i}\right)$, two severe restrictions which downgrade the quality of calculations are imposed. First, the lower limit in integral $\psi_{\beta}(\omega)$ cannot be zero because $t^{\beta-1} \rightarrow \infty$ as $t \rightarrow 0$ for $\beta<1$. Second, the upper limit must be finite $(T<\infty)$ in order to store the sampling of the mentioned functions in a computer's memory. In other words we are not using the intended limit

$$
\begin{aligned}
\tilde{f}_{\beta}(\omega) & =\lim _{T \rightarrow \infty} \int_{0}^{T} f_{\beta}(t) e^{-i \omega t} d t \\
& =\lim _{T \rightarrow \infty} \int_{-\infty}^{\infty}\{H(t)-H(t-T)\} f_{\beta}(t) e^{-i \omega t} d t
\end{aligned}
$$

instead we are calculating a member of such succession whose distance to $\tilde{f}_{\beta}(\omega)$ depends on $\beta$ and $T .^{2}$ It happens that the evaluation of the integral for fixed $\omega$ is lesser in modulus than expected, as a numerical sampling of this integral for a given $\beta$ and different $T<\infty$ shows. This situation is getting worse when $\beta \rightarrow 0^{+}$because the decaying function $e^{-t^{\beta}}$, which is integrable, tends to $e^{-1} \forall t>0$, which is not, making the erased part of the integral $\tilde{f}_{\beta}$ [i.e., $\int_{T}^{\infty} f_{\beta}(t) e^{-i \omega t} d t$ ] not negligible as $\omega \rightarrow 0^{+}{ }^{3}$ Nevertheless, being aware of this,

\footnotetext{
${ }^{2}$ Here, $f_{\beta}$ stands for $\phi_{K, \beta}(t)$ or $-\phi_{K, \beta}^{\prime}(t)$, and $\tilde{f}_{\beta}$ stands for $\chi_{\beta}$ or $\psi_{\beta}$, respectively.

${ }^{3}$ The window function, $W_{T}(t) \equiv\{H(t)-H(t-T)\}$, when Fourier transformed yields a $T \operatorname{sinc}(\omega T / 2)$ function, in modulus, of contracting width as $T \rightarrow \infty$. Its behavior is that of an identity of the
} 

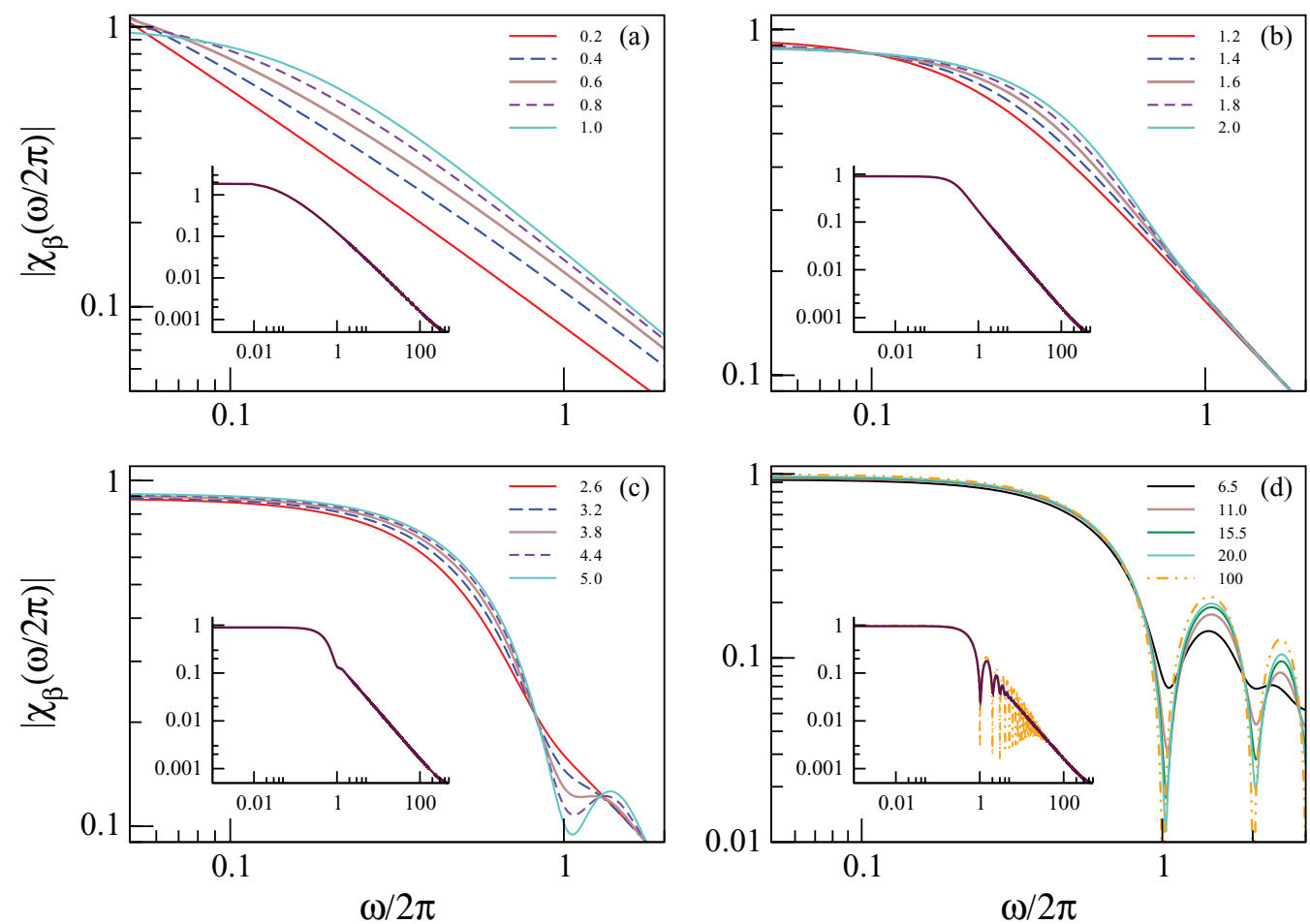

FIG. 1. (Color online) Modulus of Kohlrausch (and related functions) fast Fourier transforms for several $\beta$ values. (a) $0<\beta \leqslant 1, \delta \beta=0.2$. (b) $1.2 \leqslant \beta \leqslant 2, \delta \beta=0.2$. (c) $2.6 \leqslant \beta \leqslant 5, \delta \beta=0.6$. (d) $6.5 \leqslant \beta \leqslant 20, \delta \beta=4.5$, and $\beta=100$ (dot-dashed line). Insets: General trend, $\beta=0.5$ (a), $\beta=1.5$ (b), $\beta=3.5$ (c), and $\beta=12.5$ and (dot-dashed line) $\beta=100$ (d).

in the following sections for every $\beta>0$ we use the same $T$ and sampling $\left(t_{i+1}-t_{i}=\delta t, \forall i \in \mathbb{N}\right)$ for the sake of a homogeneous treatment and as an instrument to determine the limits of our approach as $\beta \rightarrow 0^{+}$.

However, even with this loss, we have found out that the modulus of $\chi_{\beta}$ is growing toward infinity as $\beta \rightarrow 0^{+}$at $\omega=0$. Besides it is observed from the apparent continuity of the modulus that they are decreasing monotonic functions while $\beta<1$, for low and medium frequencies. They are decreasing functions too at high frequencies because $e^{-t^{\beta}}, \beta>0$, is continuous and integrable and so the Riemann-Lebesgue [38] lemma implies the modulus of $\chi_{\beta}$ goes to zero as $\omega \rightarrow \infty$. Nevertheless this trend is restricted by the inherent periodicity and smoothness of functions in the FFT algorithm. As a consequence the Fourier transforms must be stalled in a minimum at the frontier, $\omega_{\max } \leqslant \frac{\pi}{\delta t}$, of the $\omega$ domain (see Fig. 1).

The case $\beta=1$ gives us an analytical expression for the modulus $\left|\chi_{1}(\omega)\right|=\frac{1}{\sqrt{1+\omega^{2}}}$, which explicitly shows a behavior qualitatively shared by the other modulus $\left|\chi_{\beta}(\omega)\right| \beta<1$. The slope of $\left|\chi_{1}\right|$ at $\omega=0$ is zero and decays as $\omega^{-1}$ when $\omega \rightarrow \infty$ and in $\log \omega$ scale it looks like a slow declining rounded step. For lesser values of $\beta$ numerical calculations present a similar shape with a greater height and a smaller plain as this parameter evolves from $\beta=1$ to $\beta \rightarrow 0^{+}$[see Fig. 1(a)].

convolution product, i.e., $\tilde{W}_{T} * \tilde{f}_{\beta} \stackrel{T \rightarrow \infty}{\longrightarrow} \tilde{f}_{\beta}$ when $f_{\beta} \in \mathbf{L}^{1}(\mathbb{R})$, which is not the case anymore in the limit $\beta \rightarrow 0^{+}$.
In the region $1<\beta<2$ the modulus $\left|\chi_{\beta}(0)\right|$ is still diminishing though at slower rate than in the previous one. The heights accrue, the widths enlarge, and the asymptotic tails go in the same manner as all the $\omega$ space that is available for our calculations. The curves still remain smooth without kinks or oscillations; however, the transition from plain to decaying tail becomes steeper as $\beta$ tends to 2 [see Fig. 1(b)]. By contrast at some point of the interval $2<\beta<3$ the modulus $\left|\chi_{\beta}(0)\right|$ reaches a minimum and an oscillatory profile distorts the curve progressively with the $\beta$ value. After that point the function at zero increases its value till $\left|\chi_{\infty}(0)\right|=1$, and the oscillations are developed fully along the tail [see Figs. 1(c) and 1(d)]. In detailed form, we see

$$
\chi_{\beta}(0)=\int_{0}^{\infty} e^{-t^{\beta}} d t=\Gamma\left(1+\frac{1}{\beta}\right),
$$

and $\lim _{\beta \rightarrow 0^{+}} \Gamma(1+1 / \beta)=\infty$ jointly with $\Gamma(1+1 / \beta)=1$, for $\beta=1$, and from the fact the Gamma function, $\Gamma$, interpolates as a convex function the factorial, the abovementioned trend of $\chi_{\beta}(0), \beta<1$, is justified. Moreover $\lim _{\beta \rightarrow \infty} \Gamma(1+1 / \beta)=0$ ! $=1$ so the Rolle's theorem implies an extremal point in the interval $\beta \in(1, \infty)$, which is a minimum because $\Gamma(x)>0$ and $\log \Gamma(x)$ is a convex function of $x \in(0, \infty)$. A further refinement shows the minimum is in interval $2<\beta<3$, as $\Gamma^{\prime}(1+1 / 3) / \Gamma(1+1 / 3)<0$ and $\Gamma^{\prime}(1+1 / 2) / \Gamma(1+1 / 2)>0$. A result that points to a radical change of conduct in $\chi_{\beta}$ not only at $\omega=0$. It is shown, numerically, that the decreasing with $\omega$ for each shape parameter $\beta$ is slower than in the case $\beta=2$, at low frequencies. For medium to high frequencies the modulus behavior is still of a 

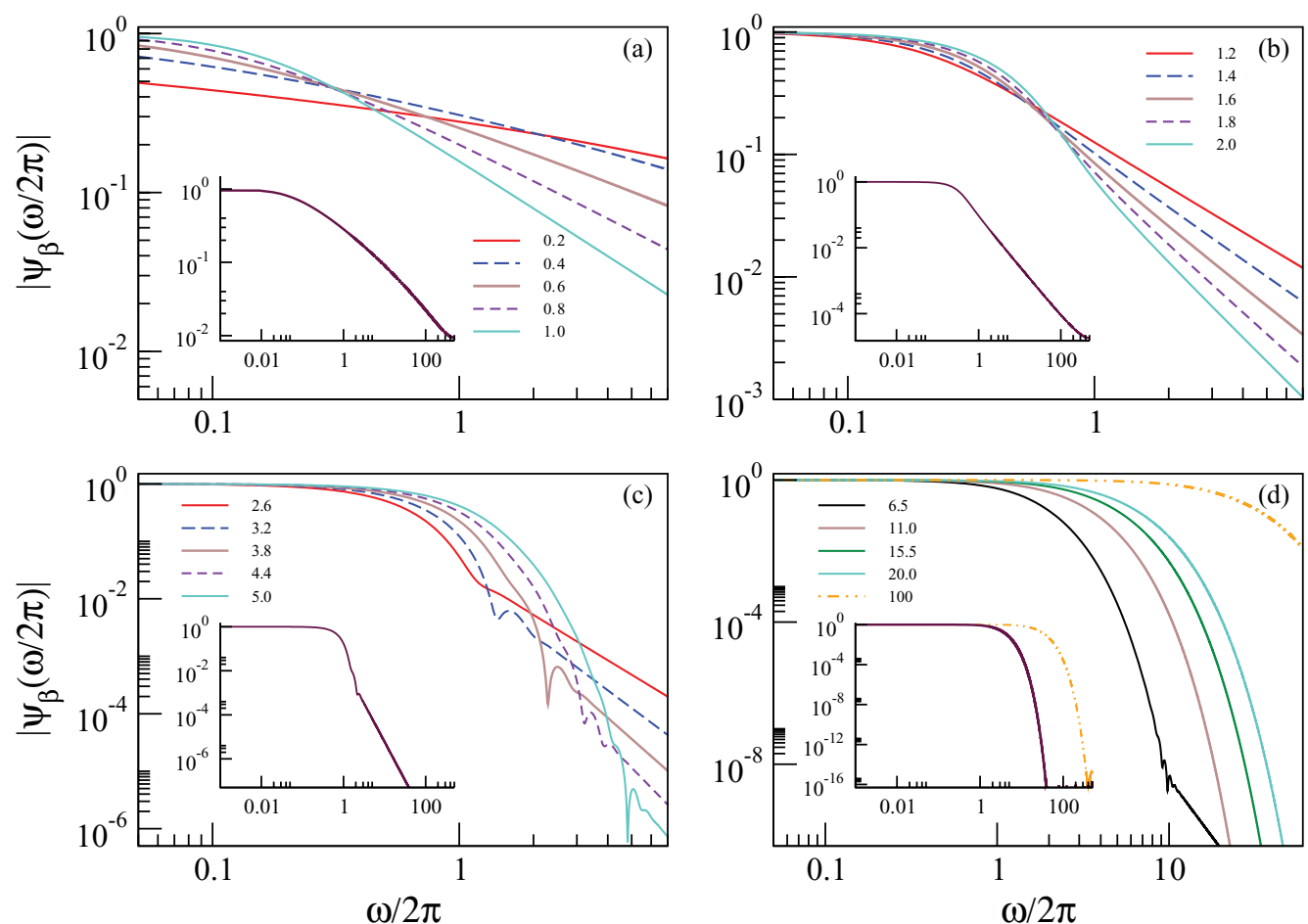

FIG. 2. (Color online) Fast Fourier transform modulus of $\beta t^{\beta-1} e^{-t^{\beta}}$ for several $\beta$ values. (a) $0<\beta \leqslant 1$, $\delta \beta=0.2$. (b) $1.2 \leqslant \beta \leqslant 2$, $\delta \beta=0.2$. (c) $2.6 \leqslant \beta \leqslant 5, \delta \beta=0.6$. (d) $6.5 \leqslant \beta \leqslant 20, \delta \beta=4.5$, and $\beta=100$ (dot-dashed line). Insets: General trend, $\beta=0.5$ (a), $\beta=1.5$ (b), $\beta=3.5$ (c), $\beta=12.5$ and (dot-dashed line) $\beta=100$ (d).

diminishing one but monotonicity does not hold anymore as the shape parameter is growing up to infinity $(3 \leqslant \beta \rightarrow \infty)$. The modulus oscillates slightly in amplitude with $\beta \approx 3$ and strongly with $\beta \rightarrow \infty$, which follows naturally since the function $e^{-t^{\beta}}$ tends to a sharp step, of width $\Delta t=1$ when $\beta \rightarrow \infty$, whose Fourier transform is a $\operatorname{sinc}(\omega)$ function (see Fig. 1).

\section{Derivative of extended exponential: $-\beta t^{\beta-1} e^{-t^{\beta}}$}

Considering $-\phi_{K, \beta}^{\prime}(t)$ in the temporal domain we see that the value at $t=0$ diverges toward infinity for $\beta<1$ and decreases monotonically to zero as $t \rightarrow \infty$, since it is the product of two monotonously decreasing functions. Letting $\beta=1$ the function starts at 1 when $t=0$ and diminishes monotonously too as it is a decreasing exponential. However if $\beta>1$ the function begins at zero and grows slowly till a maximum, whereupon it tends, overexponentially, to zero as the term $e^{-t^{\beta}}$ dominates when $t \rightarrow \infty$. With increasing $\beta \rightarrow \infty$ the maximum of the parametrized $-\phi_{K, \beta}^{\prime}$ curve grows in magnitude to infinity and the peak half-height width vanishes. This is because $t^{\beta-1} \stackrel{\beta \rightarrow \infty}{\longrightarrow} 0^{+}, \forall t<1$, and $e^{-t^{\beta}} \stackrel{\beta \rightarrow \infty}{\longrightarrow}$ $0^{+}, \forall t>1$, with the maximum at $t=1$ reaching the $\infty$ value. It resembles, not coincidentally, a Dirac $\delta$. Furthermore, we should note that $\beta t^{\beta-1} e^{-t^{\beta}}, \beta>1$, as a parametric family suffers a qualitative change of shape in the ascending slope. While $1<\beta<2$ the curve does not have inflection points on this side but has one on the descending slope $[39] ;{ }^{4}$ the

${ }^{4}$ See Appendix B. parametric family develops an inflexion point on the left side starting at $t=0$ for the limiting case $\beta=2$ which drifts toward $t \stackrel{\beta \rightarrow \infty}{\longrightarrow} 1^{-}$. This characteristic plus the distributionlike manners of the derivative for big shape parameters implies a change in modulus comportment of its Fourier transform.

A look over the FT modulus of these functions, parametrized by $\beta$ and obtained numerically, shows functions with a rounded steplike shape in $\log \omega$ scale. All of them start at the same value,

$$
\psi_{\beta}(0)=\int_{0}^{\infty} \beta t^{\beta-1} e^{-t^{\beta}} d t=1, \quad \forall \beta>0,
$$

and the "semiplateau" of the curves is width increasing. The family appears as almost layered before and after the drop of the step though with reverse order on each side of it. Nevertheless the monotonic decreasing of the modulus, only interrupted by the flattening effect at the limit of the frequency range, does not hold anymore when $\beta$ surpasses the value where $\beta t^{\beta-1} e^{-t^{\beta}}$ has two inflection points. As $\beta$ increases beyond $2, \beta>2$, attenuated fluctuations in the modulus function appear, at first as undulations, evolving to ripples until they become real damped oscillations, but with the peculiarity that their magnitude is smaller than in the case of Fourier transform of $e^{-t^{\beta}}$ when $\beta \rightarrow \infty$. Only the signal sampling and FFT numerical precision preclude this evolution, with the shape parameter, from being clearly depicted [see Fig. (2)].

We have found out, by changing the focus to $\phi_{K, \beta}^{\prime}(t)$ from $\phi_{K, \beta}(t)$ while studying their Fourier transforms, that it is possible to obtain a family bounded in all $\omega<\omega_{\max }$, with a shared origin at $\omega=0$ and with a regular behavior that is 
easily parametrizable. Moreover, for the case $0<\beta<2$ they decrease as monotonic functions of $\omega$, except for sampling (border) effects at $\omega_{\max }<\infty$.

\section{APPROXIMATION TO DERIVATIVE IN FOURIER SPACE}

A steplike behavior and the regularity of the parametric family of Fourier transformed derivatives suggest the use of a patchwork of step descending functions, selected also from a family, to approximate each of the previous ones. Many sets of functions could be candidates if only mathematical requirements were imposed, but if physical meaning is a must as well our efforts should be directed to the relaxations commonly used in scientific literature. The most general of them is the Havriliak-Negami (HN) relaxation $[9,35,40]$, and attempts have been made to link this relaxation in $\omega$ space to the Kohlrausch $(\mathrm{K})$ one in $t$ space [41]. The bridge to do this was the comparison of dielectric loss functions obtained from each decaying ( $\mathrm{K}$ or $\mathrm{HN}$ ) [41,42]. It is assumed that the functions involved admit a representation as a Laplace, or Stieltjes, transform of a non-negative function we call density, or distribution, of relaxation times, i.e.,

$$
\phi_{K, \beta}(t)=\int_{0}^{\infty} e^{-t / \tau} \rho_{K, \beta}(\tau) d \tau, \quad 0<\beta \leqslant 1,
$$

or

$$
\varphi_{\mathrm{HN}, \alpha \gamma}(\omega)=\int_{0}^{\infty} \frac{\rho_{\mathrm{HN}, \alpha \gamma}(\tau)}{1+i \omega \tau} d \tau, \quad 0<\alpha, \quad \gamma \leqslant 1,
$$

and consequently the dielectric loss will be written as

$$
-\operatorname{Im}\left(\frac{\epsilon^{*}(\omega)-\epsilon_{\infty}}{\epsilon_{0}-\epsilon_{\infty}}\right)_{K, \mathrm{HN}}=\int_{0}^{\infty} \frac{\omega \tau}{1+\omega^{2} \tau^{2}} \rho_{K, \mathrm{HN}}(\tau) d \tau .
$$

Finally, comparing a loss function of the HN type, for fixed $\alpha$ and $\gamma$, with that of the Kohlrausch type, the closest possible in response to the former, a surface of error or deviations in approximation is rendered. Moreover, a path of Kohlrausch functions as $\beta$ evolves from 0 to 1 is described in this landscape as one of minimum gradient. It is not then an equivalence among functions that is shown but rather a representation, or mapping, of one family over the other. It allows one to switch from $\omega$ space to $t$ space quickly without lengthy calculations but it is not free of uncertainty or error [43].

On the other hand it is possible to write $\chi_{\beta}(\omega)$ as a series of powers of $\frac{1}{1+(i \omega)^{\beta}}$, i.e.,

$$
\begin{aligned}
\chi_{\beta}(\omega)= & \frac{(-i) i^{\beta}}{\omega^{1-\beta}} \sum_{n=0}^{\infty} \frac{(-1)^{n}}{\left[1+(i \omega)^{\beta}\right]^{n+1}} \\
& \times\left\{\sum_{s=0}^{n}\left(\begin{array}{c}
n \\
s
\end{array}\right)(-1)^{n-s} \frac{\Gamma(\beta s+1)}{\Gamma(s+1)}\right\},
\end{aligned}
$$

and the same is true for $\psi_{\beta}(\omega)$ following Eq. (4) [15]; then it is arguable that the above-mentioned mapping of Kohlrausch functions over the Havriliak-Negami family is indeed a truncation and roundup of the original function expressed in terms of members of this family. So the next logical step to follow is to use two functions of HN type in an extended way (i.e., $\alpha$ and $\gamma$ not restricted to be smaller than 1). That construction will be a sort of "osculating plane" to a one parameter "curve" of Kohlrausch functions as $\beta$ increases up to 1 .

Being conscious of the inherent distortion the FFT is, in relationship to the analytical FT, a fit of each function $\psi_{\beta}(\omega)$-for regularity - to a "HN plane" will be done for values of $\beta$ belonging to the interval $(0.02,0.98)$ [41]. It is clear, due to periodicity of the FFT, that the approximation will fail at high frequencies in the modulus and phase. Moreover, for low $\beta$ values an equal sampling and range of points in $t$ space will not be good enough to describe the slow pace decaying of the Kohlrausch function, as it was for values of $\beta$ nearer to 1. This loss of accuracy will be reflected in Fourier space by a loss of energy in the spectrum and a major repercussion of the smoothness at the border $\omega_{\max }$ in blurring actual values of the modulus. Nevertheless we show that this approximation gives a good template at low frequencies of $\omega$ space to allow a correct identification of stretched exponentials in whole $t$ space. Such reconstructions together with their errors are presented below. In addition, gathering the $\mathrm{HN}$ parameters for all calculations gives us functions of the variable $\beta$ which allow us to calculate intermediate points of the original grid $(0.02,0.98)$. Moreover some extension to interval $1<\beta \leqslant 2$ of this picture is commented as $\exp \left(-t^{\beta}\right)$ is still the Laplace transform of a positive density [26,27,32], i.e., $\rho_{K, \beta} \geqslant 0, \forall \tau<\infty$.

It should be noted, as a technical digression, that in parallel to this scheme of changing from $t$ space to $\omega$ space to study relaxations other authors prefer to use the $\tau$ and $t$ spaces $[4,9,13,31,32,41,42,44]$ (i.e., the relaxation time density and the corresponding Laplace transform: the real time function). From a formal point of view the one-sided Fourier transform and the Laplace transform are both included in a more general one: the Laplace-Fourier transform. Both are formally the same if the variable used $(\omega$ or $1 / \tau)$ in the exponential which constitutes the kernel instead of a real one is a complex number. However it is true that specific analytical or numerical problems can deter us from employing one or the other variant. As an example, a closer look at the HN function shows the existence of a simple formula for the density of relaxation times, i.e.,

$$
\tau \rho_{\mathrm{HN}}(\tau)=\frac{1}{\pi} \frac{\sin \left[\gamma \arctan \left(\frac{\left(\tau_{o} / \tau\right)^{\alpha} \sin \pi \alpha}{1+\left(\tau_{o} / \tau\right)^{\alpha} \cos \pi \alpha}\right)\right]}{\left[1+2\left(\frac{\tau_{o}}{\tau}\right)^{\alpha} \cos \pi \alpha+\left(\frac{\tau_{o}}{\tau}\right)^{2 \alpha}\right]^{\gamma / 2}} ;
$$

nevertheless this expression is restricted to the use of parameter values $\alpha \leqslant 1$ and $\gamma \leqslant 1$. According to the limit $\alpha \rightarrow 1$ (HN tends to $\mathrm{CD}$ ), the function $\tau \rho_{\mathrm{HN}}$ loses differentiability and continuity at the maximum and a sharp cutoff of relaxation times is imposed ( $\tau \leqslant \tau_{o}$ if $\alpha=1$ ). The situation is even worse for CD relaxation as $\gamma \rightarrow 1$ since the density $\rho_{\mathrm{HN}}(\tau)$ becomes singular as a Dirac $\delta$ at $\tau_{o}$. To our knowledge no alternative or extension to this formula has been raised for the $\alpha>1$ and $\gamma>1$ cases. So, in the best scenario any approximation to the squeezed exponential, $\exp \left(-t^{\beta}\right)$, with $1<\beta \leqslant 2$, using HN-like functions should come from numerical analysis only [45].

\section{A. The dependence of HN plane with Kohlrausch shape parameter}

The function $\beta t^{\beta-1} e^{-t^{\beta}}$ is sampled from $T_{\min }=0.001$ to $T_{\max }=1000$ at intervals of $\delta t=0.001$, after which the array 
of numbers is transformed with a FFT algorithm (fftw3) to a sequence of complex numbers and their conjugates, starting at $\omega=0$ and ending at $\omega_{\max } / 2 \pi=500.0005$ at intervals of $\delta \omega / 2 \pi \approx 0.001000001$. As a numerical result from a discrete Fourier summation, whose domain interval is finite and constant, it should be noted that coefficients are given rescaled to $1 / T_{\max }$, compared with the analytically desired ones. Moreover an error is added because the limits of integration in Eq. (3) are no longer 0 and $\infty$ but are changed to $T_{\min }$ and $T_{\max }$. This can be estimated by the incomplete $\Gamma(\ldots, \cdots)$ function for null frequency. Nevertheless at the first stage we proceed with an ad hoc normalization to use regular sequences of fast Fourier transforms, with different shape parameters $\beta$, as near as possible to theoretical ones (i.e., at least starting at 1) [as deduced from Eq. (6)].

The result is the convolved function $\tilde{W}_{T} *-\tilde{\phi}_{K, \beta}^{\prime} \equiv$ $\hat{\psi}_{\beta}(\omega) \approx \psi_{\beta}(\omega)$, and a nonlinear adjustment to a couple of $\mathrm{HN}$ type relaxations is done. At this point we should mention that the canonical form of the $\mathrm{HN}$ relaxation, $\frac{1}{\left[1+(i \omega)^{\alpha}\right]^{\gamma}} \equiv \varphi_{\alpha \gamma}(\omega)$, is substituted with a frequency rescaled version, because there is no reason to consider time scale parameters in both Kohlrausch $\left(\tau_{K}=1\right)$ and Havriliak-Negami $\left(\tau_{\mathrm{HN}}\right)$ relaxations as equal. This has indeed already been proven by other authors for a one-term approximation (equivalence-of-loss), which stated $\tau_{K} / \tau_{\mathrm{HN}}$ as a nonconstant function of $\beta[41,43]$.

In short what we have is

$$
\begin{aligned}
\psi_{\beta}(\omega) & \approx \hat{\psi}_{\beta}(\omega) \simeq \lambda \varphi_{\alpha_{1} \gamma_{1} \tau_{1}}(\omega)+(1-\lambda) \varphi_{\alpha_{2} \gamma_{2} \tau_{2}}(\omega) \\
& =\frac{\lambda}{\left[1+\left(i \omega \tau_{1}\right)^{\alpha_{1}}\right]^{\gamma_{1}}}+\frac{1-\lambda}{\left[1+\left(i \omega \tau_{2}\right)^{\alpha_{2}}\right]^{\gamma_{2}}}, \quad|\lambda| \leqslant 1,
\end{aligned}
$$

a "simple" seven-parameter nonlinear setting. Therefore some previous spurious adjustments were carried out to narrow

TABLE I. Formulas used to adjust $\alpha_{i} \gamma_{i=1,2}$ data and their fitting

\begin{tabular}{|c|c|c|}
\hline Data & Adjustment & Constants \\
\hline$\alpha_{1} \gamma_{1}$ & Ad1: $m \beta+d+\exp (-E \beta+D)$ & $\begin{array}{c}m=1.090375 \\
d=-0.116589 \\
E=6.49191 \\
D=1.52956 \\
\text { Corr. } 0.996980\end{array}$ \\
\hline$\alpha_{1} \gamma_{1}$ & $\mathrm{Ad} 2: a+b \beta^{c}+B \exp \left[-C\left(\beta-\beta_{0}\right)^{2}\right]$ & $\begin{array}{c}a=0.3612 \\
b=0.613556 \\
c=1.77714 \\
B=2.72558 \\
C=19.9893 \\
\beta_{0}=0.0047320 \\
\text { Corr. } 0.987124\end{array}$ \\
\hline$\alpha_{1} \gamma_{1}$ & Ad3: $a+b \beta^{c}, 0.6 \leqslant \beta<1$ & $\begin{array}{c}a=0.36023 \\
b=0.613556 \\
c=1.77714 \\
\text { Corr. } 0.999910\end{array}$ \\
\hline$\alpha_{2} \gamma_{2}$ & Ad: $a+b \beta^{c}$ & $\begin{array}{c}a=0.279187 \\
b=0.717851 \\
c=1.51813 \\
\text { Corr. } 0.998380\end{array}$ \\
\hline
\end{tabular}
parameters. Corr. $=$ correlation coefficient of nonlinear fit. $\beta \leqslant 1$. the space parameter, thus making the search of them more simple and the dependence on $\beta$ less noisy. The first one was to consider $\left|\hat{\psi}_{\beta}(\omega)\right|^{2} \simeq \lambda\left|\varphi_{(\alpha \gamma \tau)_{1}}(\omega)\right|^{2}+(1-\lambda)\left|\varphi_{(\alpha \gamma \tau)_{2}}(\omega)\right|^{2}$. The second was to take account of the cross term, though not the angle between two vectors for a given frequency. At the end a complete fit to the square modulus of $\hat{\psi}(\omega)$ by means of the expression $\lambda^{2}\left|\varphi_{1}(\omega)\right|^{2}+(1-\lambda)^{2}\left|\varphi_{2}(\omega)\right|^{2}+2 \lambda(1-$ $\lambda)\left|\varphi_{1}(\omega)\right|\left|\varphi_{2}(\omega)\right| \cos \left\{\left[\lambda \varphi_{1} \widehat{(1-\lambda)} \varphi_{2}\right](\omega)\right\}$ was done. No relevant dependency on the imaginary part of $\lambda$ was found, whereby at this final stage it was considered real $(\operatorname{Im}(\lambda) \equiv 0)$, positive, and smaller than or equal to $1\left(1 \geqslant \lambda \in \mathbb{R}^{+}\right)$; no further adjustment to the phase equation of the system [Eq. (7)] was performed. The parameters obtained from the modulus equation were enough to fulfill the former with the aid of a first-order correction to describe numerical drop to zero at $\omega_{\max }$.

\section{B. Numerical fitting to parameters: Dependence on $\beta$}

Previously two fittings were proposed for $\alpha \gamma$ and $\tau_{K} / \tau_{\mathrm{HN}}$ when only one $\mathrm{HN}$ function was under discussion. The first was like $\alpha \gamma \approx \beta^{1.23}$ and the second was of the type $\log _{10}\left(\tau_{\mathrm{HN}} / \tau_{K}\right) \approx 2.6 \sqrt{(1-\beta)} \exp (-3 \beta)$ [41]. Both had agreement with data and seemed flexible enough to be employed as a starting point for our approximation. We define the following group of parameters to be adjusted: $\alpha_{1} \gamma_{1}, \alpha_{2} \gamma_{2}$, $\tau_{1} \tau_{2}, \tau_{1} / \tau_{2}, \alpha_{1}, \alpha_{2}$, and $\lambda$; with their fittings it is possible to reconstruct any of the original seven as a simple function of $\beta$. This effectively eliminates the ambiguity of using several parameters since they are entirely dependent on the shape

TABLE II. Formulas used to adjust $\tau_{1} \tau_{2}$ and $\tau_{1} / \tau_{2}$ data and their fitting parameters. Corr. $=$ correlation coefficient of nonlinear fit.

\begin{tabular}{|c|c|c|}
\hline Data & Adjustment & Constants \\
\hline$\tau_{1} \tau_{2}$ & Ad1: $\beta^{d}(1-\beta)^{f} \operatorname{sech}\left[M(1-\beta)^{g}\right]$ & $\begin{array}{c}d=-2.00474 \\
f=0.0955231 \\
g=3.42771 \\
M=18.6936 \\
\text { Corr. } 0.999072\end{array}$ \\
\hline$\tau_{1} \tau_{2}$ & $\operatorname{Ad} 2: \beta^{d}(1-\beta)^{f} \exp \left[M(1-\beta)^{g}\right]$ & $\begin{array}{c}d=18.9847 \\
f=0.0417098 \\
g=1.17141 \\
M=30.2978 \\
\text { Corr. } 0.998792\end{array}$ \\
\hline$\tau_{1} / \tau_{2}$ & Ad1: $\frac{A\left[1-\exp \left(-\sigma \beta^{d}\right)\right]}{1+B \exp [-M \operatorname{arctanh}(2 \beta-1)]}$ & 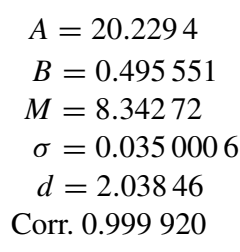 \\
\hline$\tau_{1} / \tau_{2}$ & $\mathrm{Ad} 2: \exp \left[-\exp \left(\sum_{i=0}^{i=4} a_{i} \beta^{i}\right)\right]$ & $\begin{array}{l}a_{0}=2.60006 \\
a_{1}=2.89485 \\
a_{2}=-29.9314 \\
a_{3}=43.8755 \\
a_{4}=-20.5447 \\
\text { Corr. } 0.999509\end{array}$ \\
\hline
\end{tabular}
$\beta \leqslant 1$. 
parameter. For the interval $\beta \in(0.02,1.0]$ the points calculated are at $0 . x 2,0 . x 5,0 . x 8$, or $0 . x+0.1$, with $x=0,1,2, \ldots 9$. However for $\beta \in(1.0,2.0]$ the points are sparser; we fit those of form $1 . x 5$ or $1 . y$ with $x=0,1,2, \ldots, 9$ and $y=1,2, \ldots, 9$. The functions fitting parameters in this second group are different from those in the first one, and the original $\alpha_{1,2}, \gamma_{1,2}$, $\tau_{1,2}$, and $\lambda$ are used directly instead of the mentioned ones. It should be noted that the real $\mathrm{HN}$ behavior of approximants $\varphi_{(\alpha \gamma \tau)_{1}}(\omega)$ and $\varphi_{(\alpha \gamma \tau)_{2}}(\omega)$, i.e., $\alpha_{1,2} \leqslant 1, \gamma_{1,2} \leqslant 1$, is entirely lost outside of interval $\beta \in(0.3,1.0)$. The later is contained in $(0.2,1.0)$, beyond which curvature of $\beta t^{\beta-1} e^{-t^{\beta}}$ changes its complex roots, denoted $t_{ \pm}^{\beta}$, to real ones [39]. ${ }^{5}$

Tables I, II, and III display fitting functions and their parameter values for $\alpha_{1} \gamma_{1}, \alpha_{2} \gamma_{2}, \tau_{1} \tau_{2}, \tau_{1} / \tau_{2}, \alpha_{1}, \alpha_{2}$, and $\lambda$ as functions of $\beta$. The simplest expression comes first, though, when appropriate, there are other alternatives in second place. This is the case of the $\alpha_{1} \gamma_{1}$ adjustment where three options are given. The third one picks up the product behavior as $a+b \beta^{c}$ for $\beta \in[0.6,1.0]$. This is the originally desired one, which allows one to calculate an asymptote like $m \beta+d$ when $\beta \approx 1$, both curves are subtracted to data and the remainder points are fitted to different exponential functions. By combining the exponential functions with the extracted ones we obtained the first and second fits of Table I for the product $\alpha_{1} \gamma_{1}$. Some discrepancies among them and the data are clear when $0.02 \leqslant \beta \leqslant 0.45$. An additional conformation

${ }^{5}$ See Appendix B. is attained by computing the arithmetic mean of the first two adjustments (see Fig. 3), and it deserves attention. The noise of the smallest $\beta$ values and its magnitude, and the change in the trend of data from decreasing to increasing as $\beta$ grows toward 1 , force us to employ at least two fittings in order to describe accurately the minimum of the graph. So the mean is a sort of rough weighted method which saves the trend $\sim \mathcal{O}\left(\beta^{c}\right)$ at the end of the interval and gives a good representation of the extremal point without betraying very much the rising of the curve at the $\beta$ values closest to zero. Duplicity of functions is given too for the cases $\tau_{1} \tau_{2}, \tau_{1} / \tau_{2}$, and $\lambda$, nevertheless it is not so critical as in the former example; instead it is an alternative formulation. They are not shown in graphics; they are just recorded in Tables II and III. The following two pictures represent the data and curves found: $\alpha_{1} \gamma_{1}$ in Fig. 3(a) and $\alpha_{2} \gamma_{2}$ in Fig. 3(b). In Fig. (4), $\tau_{1} \tau_{2}$ and $\tau_{1} / \tau_{2}$ are in the left panel and $\alpha_{1,2}$ and $\lambda$ are in the right panel.

The adjustment of parameters $\alpha_{1,2}, \gamma_{1,2}, \tau_{1,2}$, and $\lambda$ with $1<$ $\beta \leqslant 2$ are equally done. Nevertheless two warnings should be given. First the approximants $\varphi_{(\alpha \gamma \tau)_{1,2}}(\omega)$ are not of the HN type anymore because $\alpha_{1}>1$ and $\gamma_{1,2}>1$. However the functional form is conserved in our approximations, hence the "HN-like" designation throughout the text for $\varphi_{(\alpha \gamma \tau)_{1,2}}(\omega)$, irrespective of the $\beta$ value while it be less than 2 . The second warning is even more important: as $\beta \rightarrow 2$ the analytical Fourier transform of the Kohlrausch function, $\chi_{\beta}$, evolves to zero with a dropping of its modulus faster than in the stretched case $(\beta \leqslant 1)$. As a consequence the approach to $\psi_{\beta}(\omega)$ explained here can only

TABLE III. Formulas used to adjust $\alpha_{1}, \alpha_{2}$, and $\lambda$ data and their fitting parameters. Corr. $=$ correlation coefficient of nonlinear fit. $\beta \leqslant 1$.

\begin{tabular}{|c|c|c|}
\hline Data & Adjustment & Constants \\
\hline$\alpha_{1}$ & Ad: $\exp \left[A+B(1-\beta) \exp \left(-M\left|\beta-\beta_{0}\right|^{d}\right)\right]$ & $\begin{array}{c}A=-0.0131799 \\
B=-1.45268 \\
M=4.01763 \\
\beta_{0}=0.188585 \\
d=1.51528 \\
\text { Corr. } 0.998905\end{array}$ \\
\hline$\alpha_{2}$ & Ad: $\exp \left[A+B(1-\beta) \exp \left(-M\left|\beta-\beta_{0}\right|^{d}\right)\right]$ & $\begin{aligned} & A=0.00590101 \\
& B=-0.993309 \\
& M=7.78928 \\
& \beta_{0}=0.0549753 \\
& d=2.07124 \\
& \text { Corr. } 0.999597\end{aligned}$ \\
\hline$\lambda$ & $\mathrm{Ad} 1: \sum_{i=0}^{i=5} a_{i}(1-\beta)^{i}$ & $\begin{array}{l}a_{0}=0.0561616 \\
a_{1}=2.1984 \\
a_{2}=-1.53047 \\
a_{3}=-12.5257 \\
a_{4}=24.7067 \\
a_{5}=-12.6102 \\
\text { Corr. } 0.969331\end{array}$ \\
\hline$\lambda$ & $\mathrm{Ad} 2: \exp [M(1-\beta)] \sum_{i=0}^{i=3} a_{i}(1-\beta)^{i}, 0.18 \leqslant \beta<1$ & $\begin{array}{l}M=0.0179084 \\
a_{0}=0.0475857 \\
a_{1}=2.6703 \\
a_{2}=-6.28624 \\
a_{3}=4.3989 \\
\text { Corr. } 0.996824\end{array}$ \\
\hline
\end{tabular}



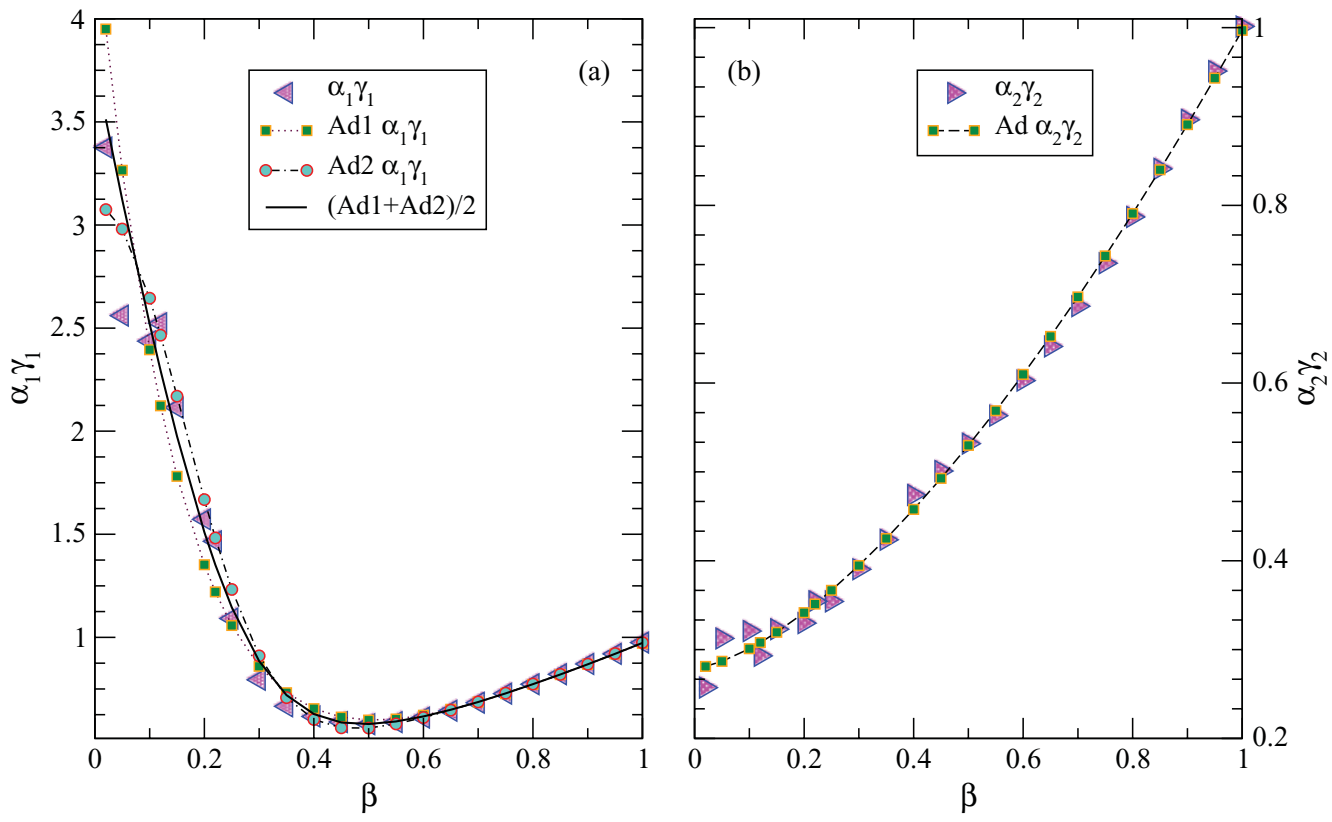

FIG. 3. (Color online) (a) Graph of $\alpha_{1} \gamma_{1}$ (left triangles) vs $\beta<1$, two analytical fits (squares with dotted line and circles with dot-dashed line) of it, and the arithmetic mean of the latter (solid line). (b) The values of $\alpha_{2} \gamma_{2}$ (right triangles) are fitted to a more simple expression (squares with dashed line). See Table I for those expressions.

describe roughly the bump (peak) of the function $-\phi_{K, \beta}^{\prime}$ and not the overexponential tail for $t \rightarrow \infty$ at $\beta>1$. Even so we consider the results in Tables IV and V, together with the graphs in Fig. 5, to be illustrative of the methodology limitations.

\section{RECONSTRUCTION OF KOHLRAUSCH FUNCTION}

At the present time our aim of obtaining a quick method to identify a precise Kohlrausch relaxation in the frequency space is reasonably fulfilled. Hence, a natural question arises, how accurate is the proposed template in relation to the
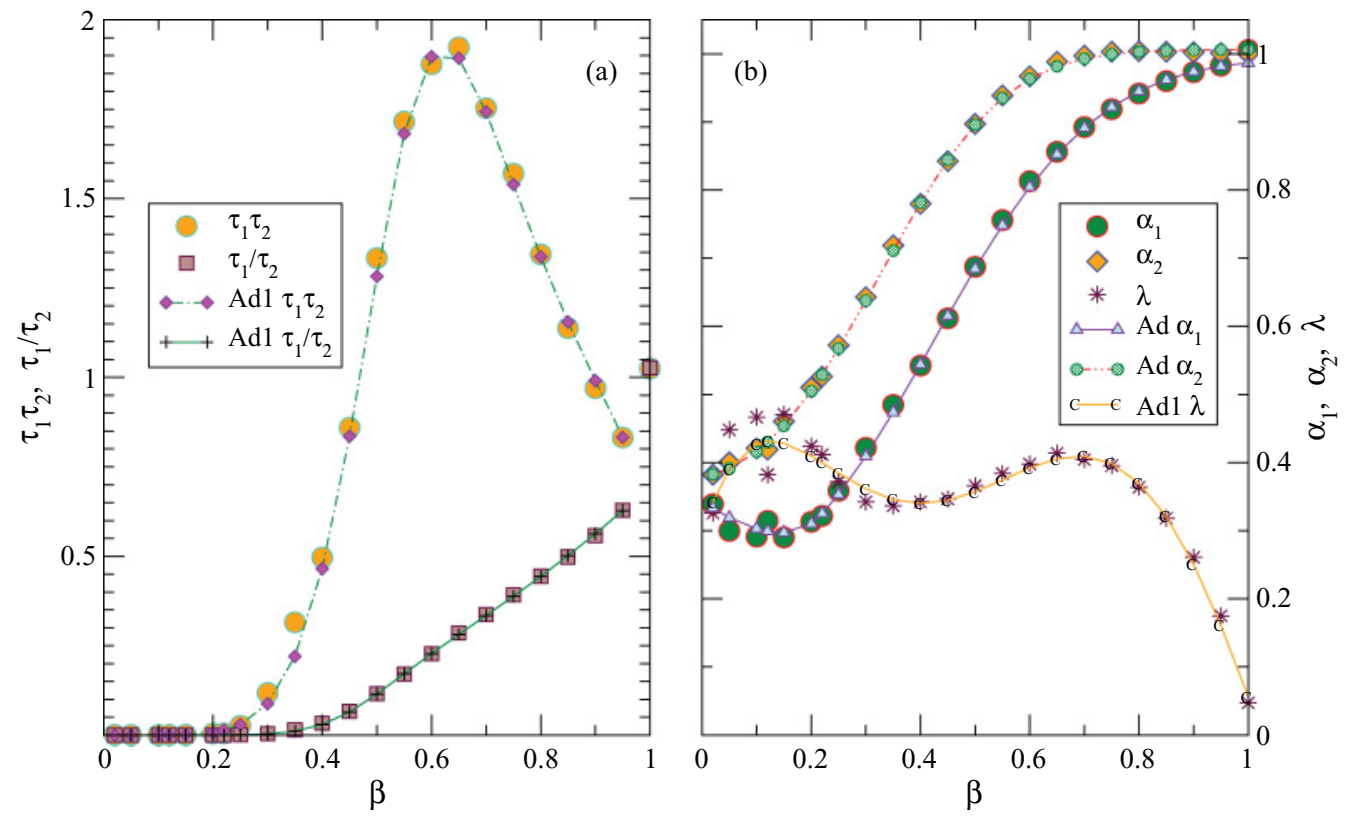

FIG. 4. (Color online) Two functions, $\tau_{1} \tau_{2}$ and $\tau_{1} / \tau_{2}$, of the frequency rescaling parameters in HN approximants are given in panel (a) (circle and square dotted lines). The best fit to each one is depicted (diamonds with dot-dashed line and cross with solid line). (b) Parameters $\alpha_{1}, \alpha_{2}$, and $\lambda$ are plotted as functions of $\beta$ (circles, diamonds, and stars, respectively). Two good adjustments to $\alpha_{1,2}$ are overimposed (small triangles with solid line and small circles with dot-dashed curve). The best possibility to adjust $\lambda$ covering all the abscissas' interval $(0,1)$ is also given [character (c) with solid line]; observe that the noisy behavior at the beginning of same precludes a better adjustment. Results are given in full detail in Tables II and III. 
TABLE IV. Fitting parameters and formulas for data $\alpha_{1,2}$ and $\gamma_{1,2}$. Corr. $=$ correlation coefficient of nonlinear fit. Data at $\beta=1.05$ are excluded from fitting, $1<\beta \leqslant 2$.

\begin{tabular}{|c|c|c|}
\hline Data & Adjustment & Constants \\
\hline$\alpha_{1}$ & Ad: $1+\left\{a_{0}(\beta-1)^{0.5}+a_{1}(\beta-1)\right\} \exp (-M \sqrt{\beta-1})$ & $\begin{array}{c}M=2.89366 \\
a_{0}=-0.198779 \\
a_{1}=1.76527 \\
\text { Corr. } 0.999957\end{array}$ \\
\hline$\alpha_{2}$ & Ad: $A+\left\{a_{0}(\beta-1)^{0.5}+a_{1}(\beta-1)\right\} \exp (-M \sqrt{\beta-1})$ & $\begin{array}{c}A=0.990982 \\
M=4.27724 \\
a_{0}=0.402462 \\
a_{1}=-1.37656 \\
\text { Corr. } 0.999750\end{array}$ \\
\hline$\gamma_{1}$ & Ad: $\{A+B \exp (C \beta)\}\left\{1+D \exp \left(-E \beta^{d}\right)\right\}$ & $\begin{array}{c}A=-0.0887192 \\
B=0.314142 \\
C=1.4697 \\
\text { Corr. } 0.999965 \\
D=0.668158 \\
E=0.783956 \\
d=8.80131 \\
\text { Corr. } 0.995870\end{array}$ \\
\hline$\gamma_{2}$ & Ad: $A \exp \left[E\left(\beta+\beta_{0}\right)^{2}\right]$ & $\begin{array}{c}A=0.307287 \\
E=0.207048 \\
\beta_{0}=1.37961 \\
\text { Corr. } 0.999991\end{array}$ \\
\hline
\end{tabular}

real spectrum? The answer is better obtained in the time domain; instead of a quadratic error measure between the two functions involved (FFT and HN approximations) we proceed to reconstruct the twofold approximant function and compare it to the original Kohlrausch one.

First of all it is necessary to know the value at $\omega=0$ of Fourier transform $\chi_{\beta}(\omega)$ for each $\beta$, because it is the only one not afforded by the closure relation (4). We could proceed tabulating numerical values of $\hat{\chi}_{\beta}(0)$ in the same manner we did

TABLE V. Fitting parameters and formulas for data $\tau_{1,2}$ and $\lambda$. Corr. $=$ correlation coefficient of nonlinear fit. Data at $\beta=1.05$ are excluded from fitting, $1<\beta \leqslant 2$.

\begin{tabular}{|c|c|c|}
\hline Data & Adjustment & Constants \\
\hline$\tau_{1}$ & Ad: $A+B \exp \left[-E\left(\beta+\beta_{0}\right)\right]$ & $\begin{array}{c}A=0.0613246 \\
B=5.1677 \\
E=1.7235 \\
\beta_{0}=0.0873948 \\
\text { Corr. } 0.999996\end{array}$ \\
\hline$\tau_{2}$ & Ad: $A+B \exp \left[-E\left(\beta+\beta_{0}\right)\right]$ & $\begin{array}{c}A=-0.0387933 \\
B=4.19896 \\
E=1.13626 \\
\beta_{0}=0.284232 \\
\text { Corr. } 0.999925\end{array}$ \\
\hline$\lambda$ & Ad: $\exp (-M \sqrt{\beta-1}) \sum_{i=0}^{i=4} b_{i}(\beta-1)^{i}$ & $\begin{array}{l}M=3.63301 \\
b_{0}=-0.249233 \\
b_{1}=8.96397 \\
b_{2}=-6.72524 \\
b_{3}=10.3182 \\
b_{4}=-3.42605 \\
\text { Corr. } 0.999862\end{array}$ \\
\hline
\end{tabular}

for $\hat{\psi}_{\beta}(0)$. This last calculation was necessary to correct the fact that the FFT as a discrete version of the Fourier integral gives a value slightly superior to the integral $\hat{\psi}_{\beta}(0)$ itself. Moreover in numerical calculations nonbounded values must be avoided, so both the FFT and Fourier integrals are given in a poorer version in which the integration (summation) lower limit is $T_{\text {min }}$ instead of zero. This means the output of FFT obtained is different than expected and a record of this disagreement is needed; we called it $t a b . \hat{\psi}_{\beta}(0)$.

Nevertheless while reconstructing $\hat{\chi}_{\beta}(\omega)$, mostly for small $\beta$ values, we observed a loss of information due to all the approximations made on $\hat{\psi}_{\beta}(\omega)$; as a consequence we opted for a mixed approach. On one hand is an analytical expression to degraded $\hat{\chi}_{\beta}(0)$, and on the other is the ratio of $\operatorname{tab} \cdot \hat{\psi}_{\beta}(0)$ to degraded $\hat{\psi}_{\beta}(0)$. The product will deliver an estimation to the desired $\chi_{\beta}(0)$ :

$$
\chi_{\beta}(0) \approx \hat{\chi}_{\beta}(0) \simeq \frac{1}{\beta} \frac{\Gamma\left(\frac{1}{\beta}, T_{\min }^{\beta}\right)-\Gamma\left(\frac{1}{\beta}, T_{\max }^{\beta}\right)}{\Gamma\left(1, T_{\min }^{\beta}\right)-\Gamma\left(1, T_{\max }^{\beta}\right)} \operatorname{tab} \cdot \hat{\psi}_{\beta}(0)
$$

with $\Gamma(\cdots, \cdots)$ being the upper incomplete Gamma function.

In Fig. 6 two reconstructions for $\beta=0.15$ and $\beta=0.85$ are shown. Each one employs the approximant obtained from Eq. (7) rescaled with $t a b . \hat{\psi}_{\beta}(0)$ in the closure relation (Eq. (4)) to estimate $\hat{\chi}_{\beta}(\omega)$. An algorithm for inverse FFT is used and an oscillating reconstructed function is achieved that follows closely the original Kohlrausch function for $t \leqslant 10$. After that moment both functions differ and the effects of a quicker decay of the stretched exponential over the twofold HN approximant function are evident. However a good agreement over the main part of the function is obtained (99.9\% for $\beta=0.85)$. 

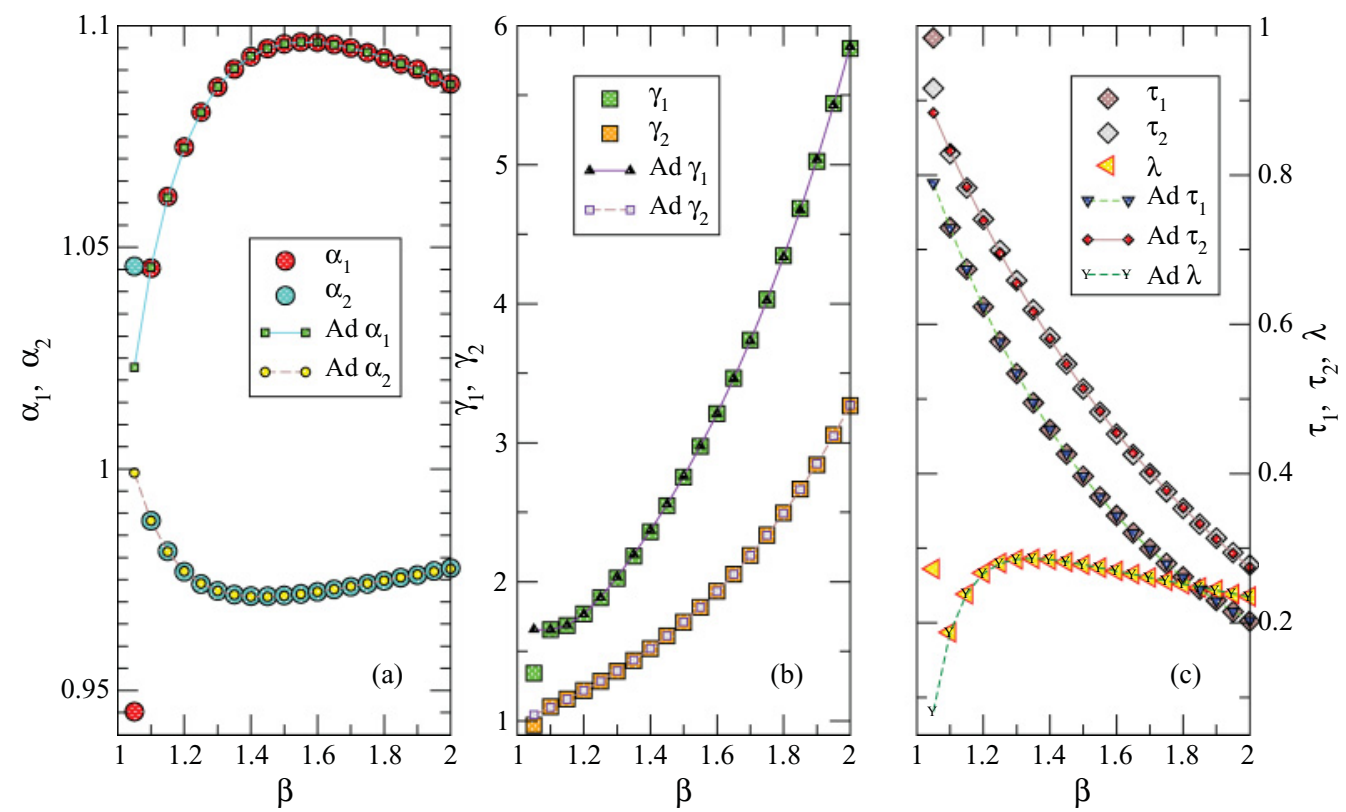

FIG. 5. (Color online) The seven parameters for HN approximants with $1<\beta \leqslant 2$ are represented. (a) $\alpha_{1}$ and $\alpha_{2}$ are shown with big circles. (b) Using squares, $\gamma_{1}$ and $\gamma_{2}$ are plotted. (c) By means of diamonds and left triangles, $\tau_{1}, \tau_{2}$, and $\lambda$ are shown. In each panel smaller symbols joined by lines adjust every HN-like parameter. The legends in the figure make clear the correspondence among data and symbols; the formulas used to obtain the fits are written in Tables IV and V.

A further correction to this $\mathrm{HN}$-like function is made when cutoff effects are introduced in the phase of such an expression. The phase of the numerical Fourier transform for $-\phi_{K, \beta}^{\prime}(t)$ goes to zero when the frequency approaches the sampling frequency (i.e., $\left.\omega \rightarrow \omega_{\max }\right)$; the phase of $\lambda \varphi_{\alpha_{1} \gamma_{1} \tau_{1}}(\omega)+(1-$ ג) $\varphi_{\alpha_{2} \gamma_{2} \tau_{2}}(\omega)$ does not. It attains asymptotically a nonzero constant value as the frequency goes to infinity, and of course is nearer to it than to zero at $\omega_{\max }$. As a consequence when inverted by fast Fourier algorithm it oscillates all the time, being the effect more visible near $t \approx 0$. Then some kind of drop off must be imposed to mimic the numerical behavior; a good choice deduced from FFT phase graphics is a linear decaying with frequency. The phase of the approximant described in Eq. (7), a function of $\omega$, will be substituted by itself
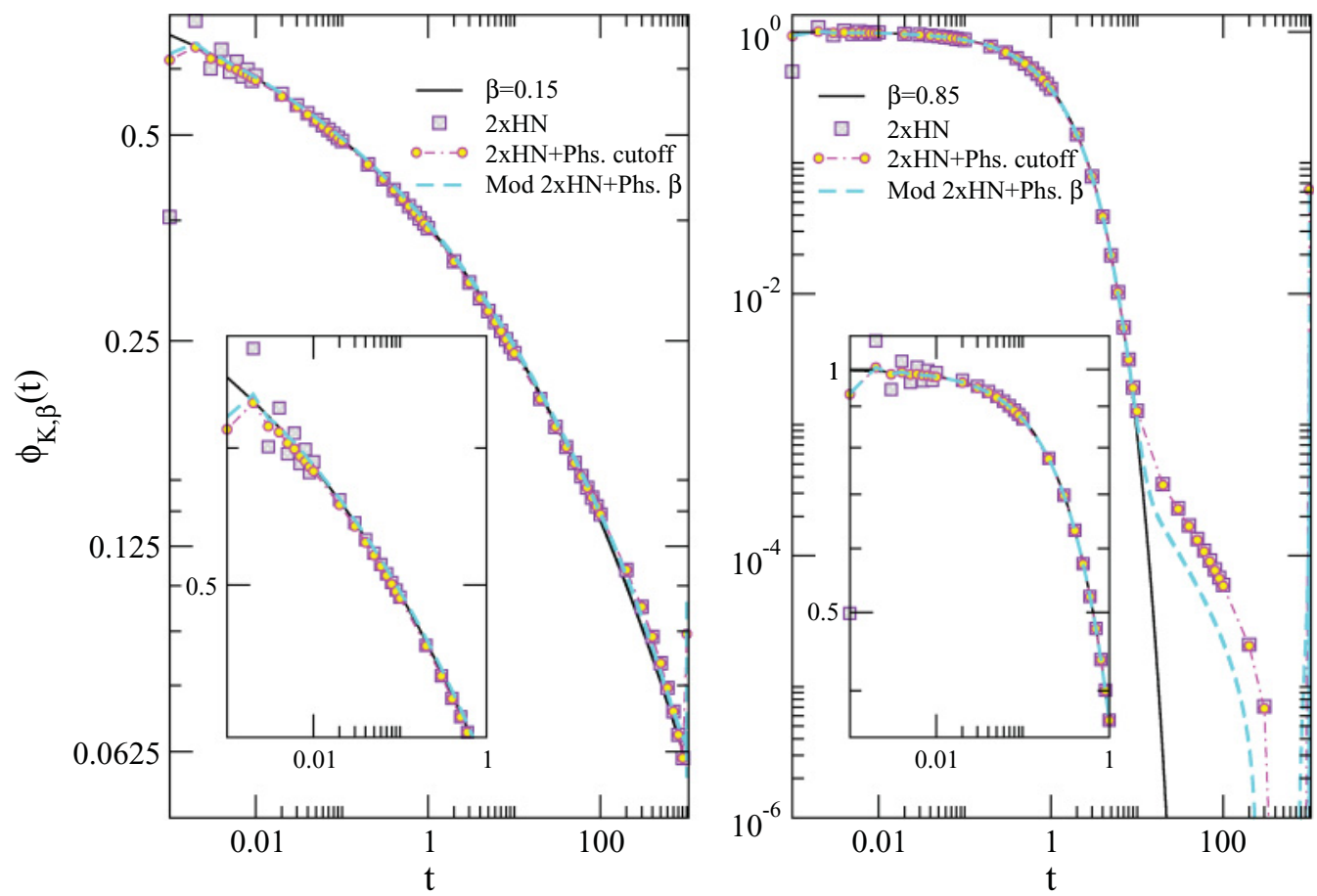

FIG. 6. (Color online) Solid line: Kohlrausch function. Squares: Twofold HN function Fourier inverted. Circles with dot-dashed line: Phase cutoff modification to previous estimator. Dotted line: Modulus of HN approximant and Kohlrausch FFT phase. 
times $\left(1-\frac{\omega}{\omega_{\max }}\right)$. The results depicted in Fig. 6 show the same trend as its predecessor but with less oscillations. Still errors and discrepancies are present surely due to the lack of accuracy in Eq. (7), because when low frequencies are involved the approximant underestimates the FFT modulus. The difference is not high, but it is enough to miss long-range information in the time domain enclosed in the phase and modulus at such frequencies (usually the first ten or so). To illustrate this point we transformed the modulus of $\chi_{\beta}(\omega)$ calculated by means of Eq. (7) and the phase obtained from FFT of $\phi_{K, \beta}(t)$ to get a third reconstruction (dotted line in Fig. 6) which continues the trend and makes a better approximation at long times to the original stretched exponential. The change in phase obtained from this swapping operation is minimal and mostly restricted to low frequencies (again less than 15 sampling points); hence it is not risky to assume the need for a third quick-fading approximant of type $\varphi_{\alpha_{3} \gamma_{3} \tau_{3}}(\omega)$ to account for the mismatch between the approximant and $\hat{\psi}_{\beta}(\omega)$ at $\omega \gtrsim 0$.

A smooth rounding of the modulus profile at high frequencies would be convenient in diminishing fluctuations at $t \approx 0$. This modification and the proposed adding of $\varphi_{\alpha_{3} \gamma_{3} \tau_{3}}(\omega)$ to the HN approximant are two possible extensions of these calculations for future works; nevertheless they are beyond the scope of the present paper.

\section{COMPARISON WITH OTHER APPROACHES AND APPLICATIONS}

\section{A. Theoretical and numerical aspects}

Once we performed the reconstruction of the Kohlrausch function from our approximation, and after considering the errors shown in the $t$ space (oscillations and long tail deviations), we presented here a comparison with available mechanisms proposed in the literature $[19,37,41,43,46]$. This allowed us to assess which computational gap would fulfill further approximants of the HN type in an infinite expansion, which would ultimately be a representation of the Fourier transform of the Kohlrausch function. In addition, it would rate its efficiency while applied to the task of identifying one or several relaxations by discriminating $\beta$ 's in $\omega$ space.

Let us start with two arbitrary approximations to $\chi_{\beta}(\omega)$, i.e., $\chi_{\beta}^{(a)}(\omega)$ and $\chi_{\beta}^{(b)}(\omega)$, we shall calculate the relative error of their modules, at frequency $\omega_{i}$, in the usual way: |1 $\left|\chi_{\beta}^{(b)}\left(\omega_{i}\right) / \chi_{\beta}^{(a)}\left(\omega_{i}\right)\right| \mid$. This function is as worthy as ||$\chi_{\beta}^{(a)}\left(\omega_{i}\right) \mid-$ $\left|\chi_{\beta}^{(b)}\left(\omega_{i}\right)\right| \mid$, the difference of modules, in evaluating the error of using one in place of the other. It has the advantage of weighting $\chi_{\beta}^{(b)}$ by $\chi_{\beta}^{(a)}$, choosing carefully the order $(a, b)$, and so yielding comparisons in the same scale of results irrespective of their magnitude, which is a useful way of presenting several segments of the spectrum and focusing on the behavior and coevolution of both functions.

Equally it is useful to define the average relative error in an interval $\left(\omega_{m}, \omega_{x}\right)$ as

$$
\left\langle\varepsilon_{\mathrm{rel}}\left(\chi_{\beta}^{(a)}, \chi_{\beta}^{(b)}, \omega_{m}, \omega_{x}\right)\right\rangle \equiv \frac{1}{\omega_{x}-\omega_{m}} \int_{\omega_{m}}^{\omega_{x}}\left|1-\frac{\left|\chi_{\beta}^{(b)}(\omega)\right|}{\left|\chi_{\beta}^{(a)}(\omega)\right|}\right| d \omega .
$$

As surrogates of $\chi_{\beta}(\omega)$ in the interval $\omega / 2 \pi \in[0,500.0005)$, we employed $\chi_{\beta}^{M}(\omega)$, calculated with the package MATHEMAT-
ICA [46], $\chi_{\beta}^{W}(\omega)$, computed with the C library described in Ref. [19], and $\chi_{\beta}^{\mathrm{FFT}}(\omega) \equiv \hat{\chi}_{\beta}(\omega)$, the FFT of $e^{-t^{\beta}}$ in the interval $t \in[0.001,1000]$ already described in this text [37]. Moreover by means of the formulas explained in Ref. [41] the substitute $\psi_{\beta}^{\mathrm{AAC}}(\omega)$ is obtained and then we arrive at $\chi_{\beta}^{\mathrm{AAC}}(\omega)$, and finally $\chi_{\beta}^{2 \mathrm{HN}}(\omega)$ is the approximation proposed in this work.

The spectrum is exhibited divided in four zones of frequencies: $\omega / 2 \pi \in$ (a) $[0,1]$, (b) $(1,10]$, (c) $(10,100]$, and (d) $(100,500]$; and the functions $\chi_{\beta}^{(*)}$ are calculated with a sampling accuracy of $\delta \omega / 2 \pi \approx 0.001000001$. Except for the case of $\chi_{\beta}^{M}$ in which four different increments have been used, namely, $\delta \omega / 2 \pi, 10 \delta \omega / 2 \pi, 100 \delta \omega / 2 \pi$, and $1000 \delta \omega / 2 \pi$ for intervals (a), (b), (c), and (d), respectively.

As MATHEMATICA comprises several well-tested routines, it is assumed that $\chi_{\beta}^{M}$ is the best approximation to $\chi_{\beta}$ obtained numerically with arbitrary precision. It serves to test the accuracy of the library in Ref. [19] which yields numbers in the float format instead of the more common format, double ; so after computing the relative error of $\chi_{\beta}^{M}$ vs $\chi_{\beta}^{W}$ the latter will be established as the numerical reference to compare with. The main tests are $\chi_{\beta}^{W}$ vs $\chi_{\beta}^{\mathrm{AAC}}$ and $\chi_{\beta}^{W}$ vs $\chi_{\beta}^{2 \mathrm{HN}}$, the relative error in second case being less, in general, than in the first one. A comparison between $\chi_{\beta}^{W}$ and $\chi_{\beta}^{\mathrm{FFT}}$ is shown as well; because both came from the same real function in the $t$ space the distortion introduced by the convolution with a finite window is set and measured. Finally $\chi_{\beta}^{\mathrm{FFT}}$ and $\chi_{\beta}^{2 \mathrm{HN}}$ are compared to reveal how much of the convolution moves us away from the ideal twofold $\mathrm{HN}$ approximation to $\chi_{\beta}$.

In Fig. 7, $\chi_{\beta}^{2 \mathrm{HN}}$ has an average relative error smaller in magnitude than that of $\chi_{\beta}^{\mathrm{AAC}}$ with respect to $\chi_{\beta}^{W}$ for every $\beta(0.1 \leqslant$ $\beta \leqslant 1$ ) inside the intervals (b), (c), and (d) mentioned above. Also observed is a good implicit filtering of the convolution effect and an adaptation to $\chi_{\beta}^{W}$ from $\chi_{\beta}^{2 \mathrm{HN}}$ and $\chi_{\beta}^{\mathrm{AAC}}$ rather than following closely $\chi_{\beta}^{\mathrm{FFT}}$ even though the smearing is present everywhere in $\omega$ and $\beta$. This situation is obviously worse when $\beta \rightarrow 0^{+}$and better when $\beta \rightarrow 1^{-}$. Naturally we can try to give up the fitting to convolved data and proceed only with numerical $\psi_{\beta}^{M, W}$, but convolution should be added anyway later while analyzing a finite temporal series in Fourier space.

Likewise the more complex approximation, $\chi_{\beta}^{2 \mathrm{HN}}$, surpasses its predecessor, $\chi_{\beta}^{\mathrm{AAC}}$, in performance in interval (a) for almost every $\beta$. The trend seems to be broken in the neighborhood of value $\beta \approx 0.60 \pm 0.05$, where the errors are similar and $\chi_{\beta}^{\mathrm{AAC}}$ overcomes the other function. Before considering this as a forbidden region, the sources of error of the twofold $\mathrm{HN}$ function as an approximant to $\psi_{\beta}$ should be taken into account. It is already commented the error inherent to the nonlinear fitting of data, and the limitations of the functional form used, to describe the few dozen low frequencies which produce large-base oscillations and misshaping of tails in $t$ space (see Fig. 6). Additionally another one has been included. We have employed mathematical expressions to adjust the seven parameters $\left\{\alpha_{1,2}, \gamma_{1,2}, \tau_{1,2}, \lambda\right\}$ as $\beta$ changes, and those functions were included in the code to generate $\chi_{\beta}^{2 \mathrm{HN}}$ (see Tables I, II, and III). By repeating the calculation of the average relative error of $\chi_{\beta}^{W}$ vs $\chi_{\beta}^{2 \mathrm{HN}}$ in the interval (a), $\omega / 2 \pi \in[0,1]$, for the original parameters $\left\{\alpha_{1,2}, \gamma_{1,2}, \tau_{1,2}, \lambda\right\}$ at $\beta=0.6$ the value $\left\langle\varepsilon_{\text {rel }}\right\rangle=2.57 \times 10^{-3}$ 

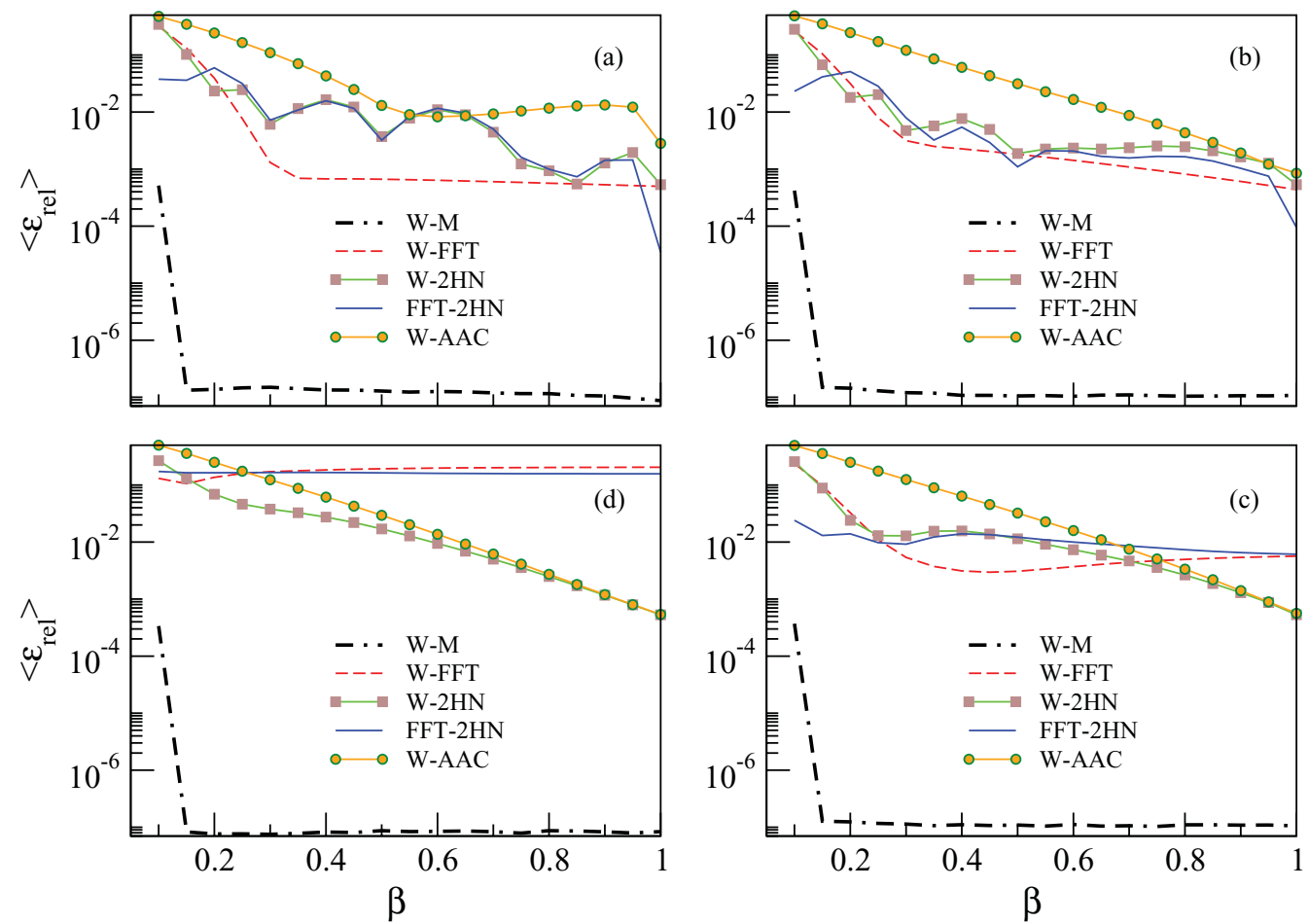

FIG. 7. (Color online) Average relative errors $\left\langle\varepsilon_{\text {rel }}\left(\chi_{\beta}^{(A)}, \chi_{\beta}^{(B)}, \omega_{m}, \omega_{x}\right)\right\rangle$ of functions $\chi_{\beta}^{(A)}$ vs $\chi_{\beta}^{(B)}$, as defined in the text, over frequency intervals: $\omega / 2 \pi \in$ (a) $[0,1]$, (b) $(1,10]$, (c) $(10,100]$, and (d) $(100,500]$. Tags (A) and (B) stand for M (MATHEMATICA, Ref. [46]), W (C library, Ref. [19]), FFT (Ref. [37]), AAC (1HN function as in Ref. [41]), and 2HN [as from Eqs. (7) and (4)].

is obtained. (The previous was $\left\langle\varepsilon_{\text {rel }}\right\rangle=1.1 \times 10^{-2}$.) And the average relative error for $\chi_{\beta}^{W}$ vs $\chi_{\beta}^{\mathrm{AAC}}$ at the same $\beta$ value is $\left\langle\varepsilon_{\text {rel }}\right\rangle=8.26 \times 10^{-3}$. Again we get a better result for $2 \mathrm{HN}$ when comparing to AAC.

One question is raised after this recalculation: Why is the relative error of $\chi_{\beta}^{2 \mathrm{HN}}$ overtaken by that of $\chi_{\beta}^{\mathrm{AAC}}$ specifically in the neighborhood of $\beta \approx 0.60$ ? A close look at the functions generating $\tau_{1} \tau_{2}$ and $\tau_{1} / \tau_{2}$ shows a maximum around $\beta \approx 0.60$ and the end of its definition at $\beta \approx 0.98$ for the first function. And the second one suffers a steep jump in the transition from $\beta=0.98$ to 1.00 as a consequence of numerical errors due to the mathematical degeneracy of formula (7) near $\beta=$ 1.00 - the Debye relaxation (see Table II and Fig. 4). As we work out $\tau_{1}$ and $\tau_{2}$ from them the propensity for numerical errors in these two intervals of $\beta$ is greater than in others ( $\beta \in$ $\{[0.55,0.65],[0.98,1.00]\})$. To correct this it is always possible to use the original values of parameters $\left\{\alpha_{1,2}, \gamma_{1,2}, \tau_{1,2}, \lambda\right\}$ and interpolate them for the intermediate values of $\beta$. Nevertheless the unifying character of the formulas for $\tau_{1}$ and $\tau_{2}$ along the interval $\beta \in(0.02,1.00]$ is lost. We can recover it with a couple of new adjustments provided we are not worried about increasing the number of parameters (constants) of such fittings. For example,

$$
\tau_{1}(\beta) \approx 2 \times 10^{-5}+A \exp \left[-M(1-\beta)^{d}\right] /(1+\beta),
$$

with $A=1.40455, M=25.8515$, and $d=4.87163$ (corr. 0.999782 ), and

$$
\begin{aligned}
\tau_{2}(\beta) \approx & 1+A(1-\beta)^{1.5} \exp \left(-M \beta^{2}\right) \\
& +B \beta^{1.5}(1-\beta) \operatorname{sech}\left(N\left|\beta_{o}-\beta\right|\right),
\end{aligned}
$$

with $A=35.7913, \quad B=18.0146, \quad M=26.7105, \quad N=$ 3.99081 , and $\beta_{o}=0.30644$ (corr. 0.999 337).

\section{B. Application to experimental data}

A main concern here is not only the use of this approximation in fitting experimental data but also the nature of the relaxation. The Kohlrausch class can be expanded to a more general type as quoted in Ref. [20]. Because they use an extended function comprising Kohlrausch and CD relaxation in glass-forming systems, the direct use of our expansion is obstructed at the present time. One obstacle is the loss of signal in convoluted data; we use $\hat{\chi}_{\beta}(\omega)$ instead of $\chi_{\beta}(\omega)$, in an attempt to compare with experimental ones which do not suffer this loss. The other handicap is more important because it is not a simple error, or uncertainty; instead it is the use of a different function with a new parameter besides the already known $\beta$. That forces a new functional expression of parameters $\left\{\alpha_{1,2}, \gamma_{1,2}, \tau_{1,2}, \lambda\right\}$ in the function of $\beta_{1}$ and $\beta_{2}$. Nevertheless we can answer two pivotal questions: whether the twofold HN is applicable here and how far in frequencies (low and high) this nonlinear regression can describe experimental data.

The relaxation proposed by Kahlau et al. [20] shows in the temporal domain a first derivative which is formally similar to that of a pure Kohlrausch function. Such a step response function is applied to mono- and bicomponent mixtures of polymers in a certain range of frequencies depending on substance and temperature. Specifically the data of propylene glycol (PG) and 2-picoline (5\%) in tristyrene (Pic-tSty) as shown in Figs. 3(a), 5, and 6 in Ref. [20] are used by those authors and by us. Once again by using the procedure 


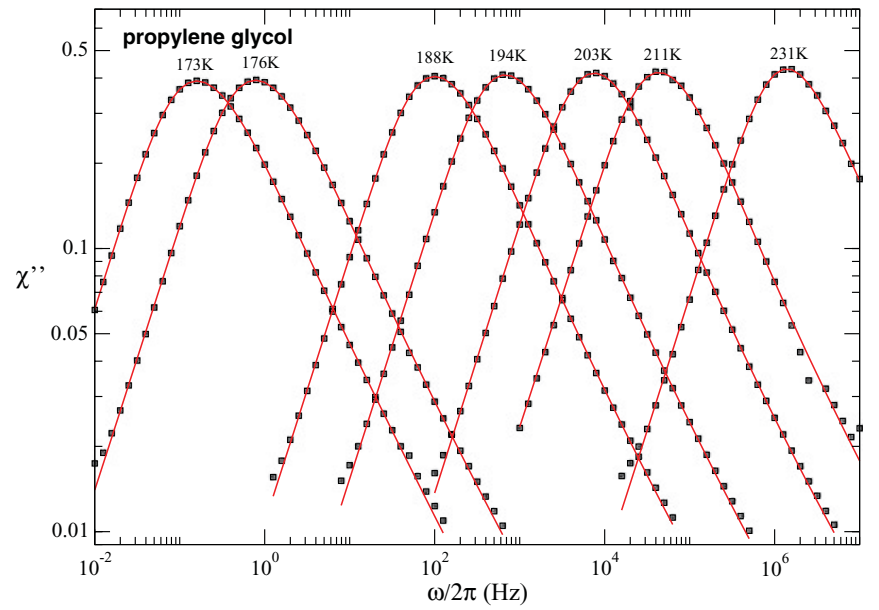

FIG. 8. (Color online) Dielectric relaxation of propylene glycol at different temperatures. Squares: Experimental data from Ref. [20]. Solid lines: Fitting to a twofold HN-like function.

of adjusting with two HN approximants the goal of the experiments is fulfilled, although this time with different parameters $\left\{\alpha_{1,2}, \gamma_{1,2}, \tau_{1,2}, \lambda\right\}$ as the objective function has changed and the data are not subject to convolution spoiling. The fittings of twofold HN functions to PG data exhibit very good agreement in the range of temperatures from $173 \mathrm{~K}$ to $231 \mathrm{~K}$ as we can see in Fig. 8. The Pic-tSty experiments (see Fig. 9) are well described too by these two (HN) functions yet we introduced a new parameter as a consequence of lack of normalization. This forces us to loosen restrictions over $\lambda$; so we first calculate a constant to describe the diminishing heights for temperatures $225 \mathrm{~K}, 230 \mathrm{~K}, 235 \mathrm{~K}$, and $240 \mathrm{~K}$ and after that the nonlinear regression is made with the constant obtained times a twofold HN approximant with condition $\lambda \leqslant 1$.

For both mixtures, mono- and bicomponents, and each temperature in the intervals of frequencies shown in Figs. 8 and 9, the agreement is really good. Only in the borders of the corresponding interval do data (crosses) and adjustments (lines) start to move away from each other. This is not a limitation of the mathematical expression used, instead it is the

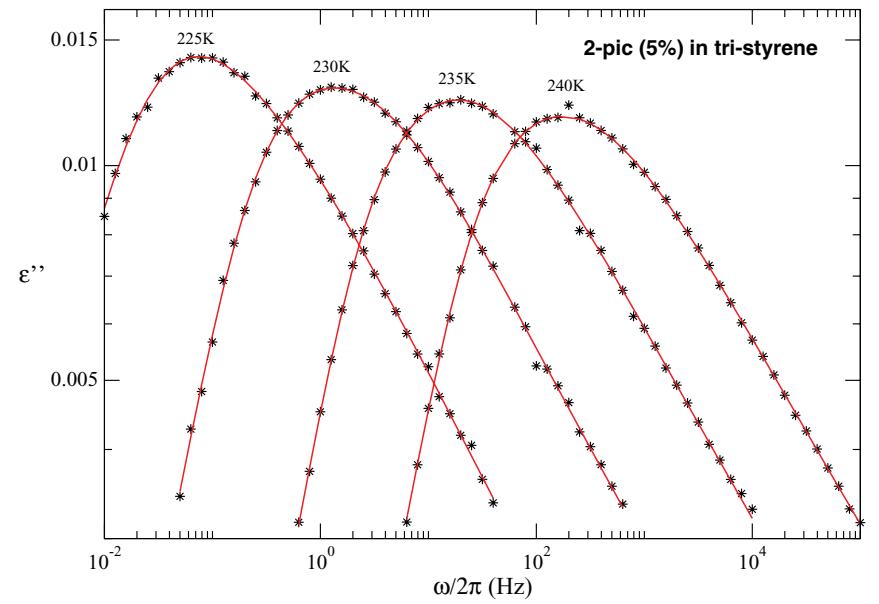

FIG. 9. (Color online) Dielectric relaxation of 2-picoline (5\%) in tristyrene at different temperatures. Stars: Experimental data from Ref. [20]. Solid lines: Fitting to a twofold HN-like function. trace of a different physical phenomenology (as, for example, the appearance of an excess wing in the high-frequency side of the interval considered).

It proves that expressions of the twofold HN-type are flexible enough to take into account dielectric relaxations of polymers subjected to a Kohlrausch-CD law (K-CD). Besides with some additional work of same type set out here, this family will serve to discriminate values of parameters $\beta_{1}$ and $\beta_{2}$ belonging to the K-CD step response function. Likewise it will let us switch between $t$ space and $\omega$ space, although some additional, and nontrivial, numerical or theoretical calculations must be arranged to account for the convolution (deconvolution) with a finite time window.

\section{CONCLUSIONS}

We qualitatively analyzed the extended exponential (stretched and squeezed), $e^{-t^{\beta}}$, and its derivative in frequency space for a wide range of shape parameters $(0<\beta \rightarrow \infty)$. The advantages and convenience of employing the Fourier transform of the derivative over the transform of the function itself, based on regularity and magnitude of modulus oscillations, are discussed. A well-known algebraic relationship between them is highlighted, releasing us from a transform other than the one of the derivative. An approximation to the latter is made by adding, in the complex plane, two functions of the HN type. The parameters of both the approximants (HN-like functions) are depicted versus the shape parameter; mathematical expressions of them as functions of $\beta$ are deduced and commented upon. The same procedure beyond the Kohlrausch function $(0<\beta<1)$ is repeated, and equivalent graphs and adjustments are drawn too for the interval $1<\beta \leqslant 2$. Expected and observed limitations of this range of extension are briefly pointed out.

Reconstruction in the $t$ domain of twofold HN approximations were carried out for two extreme examples, $\beta=0.15$ and $\beta=0.85$; their errors relative to the original stretched exponentials are shown. Suggestions for the improvement of this approximant with another HN-like term are given. They are based on reconstructed function behavior, while modifications in phase were added to twofold $\mathrm{HN}$ functions, and on the discrepancies at low frequency between approximant and primal FFT data.

Moreover numerical comparisons show that an expansion in HN-like functions is a promising way to describe complex relaxations in Fourier space. In this sense excellent agreement is found between experimental data and their fittings using twofold $\mathrm{HN}$ approximants.

In the end we obtained a method to switch from the $t$ domain to the $\omega$ domain that is quicker than an exhaustive numerical calculation. Though spoiled with convolution effects, it endows us with a template, simple and efficient, to identify spectral decay as coming from a precise step function, $e^{-t^{\beta}}$, with fixed $\beta$.

\section{ACKNOWLEDGMENTS}

We would like to thank the authors of Ref. [20] (R. Kahlau and E. A. Rössler) for providing us with the $\alpha$-relaxation data 
of the polymers depicted in Figs. 8 and 9. We also thank Centro de Calculo (IFF), CTI (CSIC), and CESGA for allocation of computer time. This work has been supported by DGICYT, Spain, Grant No. FIS2010-18132.

\section{APPENDIX A}

Usually Kohlrausch functions are defined with two parameters instead of one. The shape parameter $\beta$ and the time scale factor $\tau_{K}$ are combined to give the form $\phi_{\beta, \tau_{K}}(t)=$ $\exp \left[-\left(t / \tau_{K}\right)^{\beta}\right]$, which is most commonly found in literature that we used, i.e., $\phi_{K, \beta}(t)$. However to calculate one sidedFourier transform of the former the knowledge of the latter is quite enough to describe it, if frequency domain is rescaled with $\tau_{K}$. This is because

$$
\begin{aligned}
\mathcal{L}_{i}\left\{\phi_{\beta, \tau_{K}}\right\}(\omega) & \equiv \int_{0}^{\infty} e^{-\left(t / \tau_{K}\right)^{\beta}} e^{-i \omega t} d t \\
& =\tau_{K} \int_{0}^{\infty} e^{-u^{\beta}} e^{-i \omega \tau_{K} u} d u \equiv \tau_{K} \mathcal{L}_{i}\left\{\phi_{K, \beta}\right\}\left(\omega \tau_{K}\right),
\end{aligned}
$$

with $u=\frac{t}{\tau_{K}}$. And equally, the Fourier transform of the derivative is expressed by the aid of $\mathcal{L}_{i}\left\{\phi_{K, \beta}^{\prime}\right\}$ as

$$
\begin{aligned}
\mathcal{L}_{i}\left\{\frac{d}{d t} \phi_{\beta, \tau_{K}}\right\}(\omega) & \equiv \frac{-\beta}{\tau_{K}} \int_{0}^{\infty}\left(\frac{t}{\tau_{K}}\right)^{\beta-1} e^{-\left(t / \tau_{K}\right)^{\beta}} e^{-i \omega t} d t \\
& =-\beta \int_{0}^{\infty} u^{\beta-1} e^{-u^{\beta}} e^{-i \omega \tau_{K} u} d u \\
& =\mathcal{L}_{i}\left\{\frac{d}{d u} \phi_{K, \beta}\right\}\left(\omega \tau_{K}\right) .
\end{aligned}
$$

\section{APPENDIX B}

We have

$$
\frac{d}{d t}\left(\beta t^{\beta-1} e^{-t^{\beta}}\right)=\beta t^{\beta-2} e^{-t^{\beta}}\left\{(\beta-1)-\beta t^{\beta}\right\},
$$

giving a maximum of $-\phi_{K, \beta}^{\prime}(t)$ at $t_{*}=(1-1 / \beta)^{1 / \beta} \stackrel{\beta \rightarrow \infty}{\longrightarrow} 1^{-}$, for $\beta>1$. Also the derivative function, $\frac{d}{d t}-\phi_{K, \beta}^{\prime}(t)$, has zero value at $t=0$ if $\beta>2$, but this implies a zero in the second derivative by the Rolle's theorem, i.e., an inflection point in the interval $\left(0, t_{*}\right) \subseteq(0,1)$. On the other hand

$$
\begin{aligned}
\left(\beta t^{\beta-1} e^{-t^{\beta}}\right)^{\prime \prime}= & \beta t^{\beta-3} e^{-t^{\beta}}\{(\beta-1)(\beta-2) \\
& \left.-3 \beta(\beta-1) t^{\beta}+\beta^{2} t^{2 \beta}\right\}=0,
\end{aligned}
$$

if any of three members is zero and $\beta \neq 1$. If $1<\beta<2$ the first term is always positive, so the second term, in the interval $t \in(0, \infty)$, and only when $t \rightarrow \infty$, is the product $t^{\beta-3} e^{-t^{\beta}} \rightarrow$ $0^{+}$. Therefore the inflection points will be given by the zeros of a second-order polynomial in $t^{\beta}$. The roots are

$$
t_{ \pm}^{\beta}=\frac{1}{2 \beta}\left[3(\beta-1) \pm \sqrt{9(\beta-1)^{2}-4(\beta-1)(\beta-2)}\right],
$$

i.e., one positive and one negative. The latter is discarded because $t \geqslant 0$. Only the positive one remains, which with a trivial calculation is identified as greater than $t_{*}$ or recognized as such as a consequence of Rolle's theorem, given that $\frac{d}{d t}-$ $\phi_{K, \beta}^{\prime}(t)=0$ at $t=t_{*}$ and $t \rightarrow \infty$. As a consequence there is not an inflection point inside the interval $\left(0, t_{*}\right)$ while $1<\beta<$ 2 . In short, the root minus of $\{\cdots\}$, i.e., $t_{-}^{\beta}$, crossing from the negative part of the real line, $(1<\beta<2)$, to the positive side, $(\beta>2)$, marks at $\beta=2$ the occurrence of an inflexion point to the left of maximum $t_{*}$ for the function $-\phi_{K, \beta}^{\prime}(t)$.
[1] R. Kohlrausch, Pogg. Ann. Phys. Chem. 91, 56 (1854); 91, 179 (1854).

[2] G. D. J. Phillies and P. Peczak, Macromolecules 21, 214 (1988).

[3] J. C. Phillips, Rep. Prog. Phys. 59, 1133 (1996).

[4] M. N. Berberan-Santos, E. N. Bodunov, and B. Valeur, Chem. Phys. 315, 171 (2005).

[5] R. S. Anderssen, S. A. Husain, and R. J. Loy, ANZIAM J. (E) 45, C800 (2004).

[6] P. Debye, Ver. Deut. Phys. Gesell. 15, 777 (1913).

[7] K. S. Cole and R. H. Cole, J. Phys. Chem. 9, 341 (1941).

[8] D. W. Davidson and R. H. Cole, J. Chem. Phys. 19, 1484 (1951).

[9] S. Havriliak and S. Negami, Polymer 8, 161 (1967).

[10] A. Wintner, Duke Math. J. 8, 678 (1941).

[11] G. Williams and D. C. Watts, Trans. Faraday Soc. 66, 80 (1970).

[12] G. Williams, D. C. Watts, S. B. Dev, and A. M. North, Trans. Faraday Soc. 67, 1323 (1971).

[13] C. P. Lindsey and G. D. Patterson, J. Chem. Phys. 73, 3348 (1980).

[14] E. W. Montroll and J. T. Bendler, J. Stat. Phys. 34, 129 (1984).

[15] G. H. Weiss, M. Dishon, A. M. Long, J. T. Bendler, A. A. Jones, P. T. Inglefield, and A. Bandis, Polymer 35, 1880 (1994).

[16] E. Helfand, J. Chem. Phys. 78, 1931 (1983).
[17] M. Dishon, G. H. Weiss, and J. T. Bendler, J. Res. Natl. Bur. Stand. 90, 27 (1985).

[18] R. Ferguson, V. Arrighi, J. J. McEwen, S. Gagliardi, and A. Triolo, J. Macromol. Sci. Part B: Phys. 45, 1065 (2006).

[19] J. Wuttke, e-print arXiv:0911.4796.

[20] R. Kahlau, D. Kruk, Th. Blochowicz, V. N. Novikov, and E. A. Rössler, J. Phys.: Codens. Matter 22, 365101 (2010).

[21] S. Havriliak Jr. and S. J. Havriliak, Polymer 36, 2675 (1995).

[22] R. Hilfer, Phys. Rev. E 65, 061510 (2002).

[23] M. N. Berberan-Santos, E. N. Bodunov, and B. Valeur, Chem. Phys. 317, 57 (2005).

[24] R. G. Palmer, D. L. Stein, E. Abrahams, and P. W. Anderson, Phys. Rev. Lett. 53, 958 (1984).

[25] M. F. Shlesinger and E. W. Montroll, Proc. Natl. Acad. Sci. 81, 1280 (1984).

[26] S. Bochner, Duke Math. J. 3, 488 (1937).

[27] S. Bochner, Duke Math. J. 3, 726 (1937).

[28] G. Doetsch, Theorie und Anwendung der LaplaceTransformationen (Dover, New York, 1943).

[29] D. V. Widder, The Laplace Transform (Dover, New York, 2010).

[30] P. Humbert, Bull. Sci. Math. 69, 121 (1945).

[31] H. Pollard, Bull. Am. Math. Soc. 52, 908 (1946).

[32] H. Bergstrom, Ark. Mat. 2, 375 (1952). 
[33] H. S. Wall, Analytic Theory of Continued Fractions (Van Nostrand, New York, 1948); H. S. Wall, Analytic Theory of Continued Fractions (Chelsea, New York, 1967).

[34] E. C. Titchmarsh, Introduction to the Theory of Fourier Integrals (Clarendon Press, Oxford, 1948).

[35] S. Havriliak Jr. and S. J. Havriliak, Polymer 37, 4107 (1996).

[36] J. G. Powles, J. Mol. Liq. 56, 35 (1993).

[37] M. Frigo and S. G. Johnson [http://www.fftw.org].

[38] T. M. Apostol, Mathematical Analysis (Addison-Wesley, Reading, MA, 1974).

[39] H. Rinne, The Weibull Distribution: A Handbook (CRC Press, Boca Raton, FL, 2009).
[40] S. Havriliak and S. Negami, J. Polym. Sci. Part C 14, 99 (1966).

[41] F. Alvarez, A. Alegria, and J. Colmenero, Phys. Rev. B 44, 7306 (1991).

[42] Y. Imanishi, K. Adachi, and T. Kotaka, J. Chem. Phys. 89, 7593 (1988).

[43] F. Alvarez, A. Alegria, and J. Colmenero, Phys. Rev. B 47, 125 (1993).

[44] C. Hansen, R. Richert, and E. W. Fischer, J. Non-Cryst. Solids 215, 293 (1997).

[45] H. Schafer, E. Sternin, R. Stannarius, M. Arndt, and F. Kremer, Phys. Rev. Lett. 76, 2177 (1996).

[46] Wolfram Research [http://www.wolfram.com]. 